TRANSACTIONS OF THE

AMERICAN MATHEMATICAL SOCIETY

Volume 313, Number 2, June 1989

\title{
STABLE PROCESSES WITH DRIFT ON THE LINE
}

\author{
SIDNEY C. PORT
}

\begin{abstract}
The stable processes on the line having a drift are investigated. Except for the symmetric Cauchy processes with drift these are all transient and points are nonpolar sets. Explicit information about the potential kernel is obtained and this is used to obtain specific results about hitting times and places for various sets.
\end{abstract}

\section{Statement OF Results}

Let $\tilde{X}_{t}$ be a process with stationary independent increments having log characteristic function

$$
-t|\theta|^{\alpha}(1-i h \operatorname{sgn}(\theta))
$$

where $0<\alpha<1$ or $1<\alpha \leq 2, h=\beta \tan (\pi \alpha / 2)$, and $|\beta| \leq 1$. Such processes are called drift free strictly stable processes. The processes with log characteristic functions

$$
-t|\theta|\left(1+i \frac{2 \beta}{\pi} \operatorname{sgn}(\theta) \ln |\theta|\right)
$$

are called drift free Cauchy processes (or stable processes with index $\alpha=1$ ). If $X_{t}=\widetilde{X}_{t}-b t$ we say $X_{t}$ is a stable process with drift $-b$. In this paper we will always take $b>0$.

There is an enormous literature on the behavior of drift free stable processes. Surprisingly, little has been written on stable processes with drift. In view of the extensive results known for the potential theory [6] and the path behavior $[3,4]$ for infinitely divisible processes in general, the interest today in special processes such as stable processes with drift lies in the fact that for such processes rather explicit results may be obtained.

The incorporation of a drift term changes the behavior of the process as compared to a drift free process. However the drift acts substantially different for processes with index $\alpha<1, \alpha=1$, and $\alpha>1$. In all cases except for $\alpha=1$ and $\beta=0$ (i.e. the symmetric Cauchy process) the processes with drift are transient and hit points with positive probability. This fact follows from the general theory [3 and 6]. This is in contrast to the drift free processes. For drift free processes if $\alpha<1$ the processes are transient but do not hit points,

Received by the editors July 31, 1986 and, in revised form, February 1, 1988.

1980 Mathematics Subject Classification (1985 Revision). Primary 60J30; Secondary 60J45.

Key words and phrases. Stable processes, stable processes with drift. 
while for $\alpha>1$ the processes are recurrent. General theory [3] again tells us that for $\alpha<1$ a point is irregular for itself while for $\alpha>1$ a point is regular for itself. For $\alpha=1$ and $\beta=0$ the process with drift is still recurrent and fails to hit points, while for $\beta \neq 0$ these processes are transient and hit points with positive probability.

The processes with $\beta= \pm 1$ are called strictly asymmetric. As written here $\beta=1$ corresponds to a process that can only jump to the right while $\beta=-1$ yields a process that can only jump to the left.

For each $t, X_{t}$ has a bounded continuous density $p(t, x)$. We will first discuss a process with index $\alpha \neq 1$. The quantity

$$
g(x)=\int_{0}^{\infty} p(t, x) d t
$$

is called the potential kernel for the process $X_{t}$. Let $f$ be the density of $\widetilde{X}_{1}$. Then

$$
p(t, x)=t^{-1 / \alpha} f\left(t^{-1 / \alpha}(x+b t)\right)
$$

and

$$
g(x)=\int_{0}^{\infty} t^{-1 / \alpha} f\left(t^{-1 / \alpha}(x+b t)\right) d t .
$$

For $\alpha<1$ the drift free process is also transient. Let $\tilde{g}(x)$ denote its potential kernel. This may be explicitly computed. It is given by

$$
\tilde{g}(x)=[1+\beta \operatorname{sgn}(x)]|x|^{\alpha-1}\left(1+h^{2}\right)^{-1} \frac{\Gamma(1-\alpha)}{\pi} \sin \left(\frac{\alpha \pi}{2}\right) .
$$

It is a classical result that $g(x)$ for the processes with $\alpha=2$ (Brownian motion with variance $2 t$ and mean $-b$ ) is given by $g(x)=\frac{1}{b} e^{-b x}, x>0$, and $g(x)=\frac{1}{b}, x \leq 0$. Hence forth we will exclude this process and understand processes with $\alpha>1$ to mean $\alpha<2$.

It is not possible to explicitly compute $g(x)$ for the processes with $\alpha<2$. Nonetheless we can determine some rather explicit facts about $g(x)$. General theory [3] shows that $g$ is bounded, and continuous at all $x \neq 0$. For $\alpha>1$, $g$ is continuous at 0 and $g$ has a maximum at 0 . For $\alpha<1, g$ has a jump at $0 ; g(0)=g(0+)$ and $g\left(0_{-}\right) \geq g(x)$ for all $x$. Let $h(x)$ be the probability that the process starting at 0 hits $x$. Then general theory [3] shows $h(x)=C g(x)$ where $C=g\left(0_{-}\right)^{-1}$.

Theorem 1. Let

$$
p=\frac{1}{2}+\frac{1}{\pi \alpha} \tan ^{-1}\left(\beta \tan \left(\frac{\pi \alpha}{2}\right)\right) .
$$

Then for $\alpha<1$,

$$
\begin{aligned}
g(0) & =g(0+)=\frac{1}{b} \frac{\alpha p}{1-\alpha}, \quad g(0-)=\frac{1}{b}\left[1+\frac{\alpha p}{1-\alpha}\right], \\
C & =\frac{(1-\alpha) b}{1-\alpha(1-p)}, \quad h(0)=\frac{\alpha p}{1-\alpha(1-p)} .
\end{aligned}
$$


Remark. It is interesting that the probability $h(0)$ does not depend on the magnitude of $b$. As pointed out to me by Pat Fitzsimmons, the referee, and others, this fact follows at once from the scaling property of $\widetilde{X}_{t}$, and the fact that

$$
h(0)=P\left(\widetilde{X}_{t}=b t \text { for some } t>0\right) .
$$

Indeed, for any $c>0$ and $\tilde{X}(0)=0$,

$$
\begin{aligned}
\inf \{t>0: \tilde{X}(t)=b t\} & =\inf \left\{t>0: x^{-1 / \alpha} \tilde{X}(c t)=b t\right\} \\
& =\frac{1}{c} \inf \left\{u: \widetilde{X}(u)=c^{(\alpha-1) / \alpha} b u\right\} .
\end{aligned}
$$

Taking $c^{-1}=b^{\alpha / 1-\alpha}$ we find

$$
b^{\alpha / 1-\alpha} \inf \{t>0: \widetilde{X}(t)=t\}=\inf \{t>0: \widetilde{X}(t)=b t\} .
$$

Thus $P(\tilde{X}(t)=b t$ for some $t>0)=P(\tilde{X}(t)=t$ for some $t)$.

Theorem 2. Let $\alpha<1$ and $\beta=1$. Set $\gamma=[b \cos (\pi \alpha / 2)]^{-1 /(1-\alpha)}$. Then

$$
h(-x)=e^{-\gamma x}, \quad x>0 \text {, }
$$

and

$$
g(-x)=[(1-\alpha) b]^{-1} e^{-\gamma x}, \quad x>0 .
$$

We will next determine the asymptotic behavior of $g(x)$ as $x \rightarrow \pm \infty$. Surprisingly, this can be done rather exactly. The asymptotic behavior as $x \rightarrow \pm 0$ will be given in Theorems 14 and 15 .

Theorem 3. Let $\alpha<1$. For any $n=0,1, \ldots$

$$
g(x)=\sum_{j=0}^{n} \gamma_{j}^{+}(\alpha, \beta) b^{j} x^{(j+1)(\alpha-1)}+o\left(x^{(n+1)(\alpha-1)}\right), \quad x \rightarrow \infty,
$$

and

$$
g(-x)=\sum_{j=0}^{n} \gamma_{j}^{-}(\alpha, \beta) b^{j} x^{(j+1)(\alpha-1)}+o\left(x^{(n+1)(\alpha-1)}\right), \quad x \rightarrow \infty .
$$

The coefficient $\gamma_{n}^{-}(\alpha, \beta)=\gamma_{n}^{+}(\alpha,-\beta)$ and

$$
\begin{aligned}
\gamma_{n}^{+}(\alpha, \beta)= & \frac{\alpha}{n !} \int_{0}^{\infty} f^{(n)}(u) u^{n-(n+1) \alpha} d u \\
= & \Gamma((n+1)(1-\alpha))(-1)^{n}(\pi)^{-1}\left(1+h^{2}\right)^{-(n+1) / 2} \\
& \times \sin \left[(n+1)\left(\frac{\alpha \pi}{2}+\tan ^{-1}(h)\right)\right] .
\end{aligned}
$$

For $k>1$ and $0<\frac{1}{k} x \leq y \leq k x$,

$$
g(x)-g(y) \sim \gamma_{0}^{+}(\alpha, \beta)\left[x^{\alpha-1}-y^{\alpha-1}\right], \quad x, y \rightarrow \infty,
$$

and

$$
g(-x)-g(-y) \sim \gamma_{0}^{-}(\alpha, \beta)\left[x^{\alpha-1}-y^{\alpha-1}\right], \quad x, y \rightarrow \infty .
$$


For $x>0, g(x)$ is decreasing and $g(-x)$ is asymptotically strictly decreasing.

The asymptotic expansion of $g(x)$ for $x>0$ is only of interest for $\beta>-1$ since all the coefficients for $\beta=-1$ are 0 . Similarly the expansion of $g(x)$ for $x<0$ is only of interest for $\beta<1$. Note that $g(x) \sim \tilde{g}(x), x \rightarrow \pm \infty$, and that we can express the asymptotic relationships in (1.5) by saying for $\frac{1}{k}|x| \leq|y| \leq k|x|$

$$
g(x)-g(y) \sim \tilde{g}(x)-\tilde{g}(y), \quad x, y \rightarrow \infty \pm \infty .
$$

Let $B$ be a bounded Borel set and let $T_{B}=\inf \left\{t>0: X_{t} \in B\right\}(=\infty$ if $X_{t} \notin B$ for all $\left.t>0\right)$ be the first hitting time of $B$. Let $W_{B}$, defined on $\left[T_{B}<\infty\right]$ by $W_{B}=\sup \left\{t>0: X_{t} \in B\right\}$, be the last hitting time of $B$. The process $-X_{t}$ is called the dual process to $X_{t}$. Quantities referring to the dual process are called co- and denoted by $\bumpeq$. Thus $\widehat{T}_{B}$ is the first hitting time of $B$ by the dual process. General theory shows there are unique measures $\mu_{B}$ and $\hat{\mu}_{B}$ supported on $\bar{B}$ such that

$$
P_{x}\left(T_{B}<\infty\right)=\int g(y-x) \hat{\mu}_{B}(d y) \text {, and } \widehat{P}_{x}\left(T_{B}<\infty\right)=\int g(x-y) \mu_{B}(d y) \text {. }
$$

These measures are called the co-capacitory and capacitory measure of $B$ respectively. They have a common total mass $C(B)$ called the capacity of $B$. For a point the capacity is $C=b(1-\alpha) / 1-\alpha(1-p)$.

In the sequel we will say a sequence of measures $\gamma_{x}(d y) \rightarrow \gamma(d y)$ to mean $\gamma_{x}(B) \rightarrow \gamma(B)$ weakly. The measure $e_{d}(B)=1_{B}(d)$ is the unit mass at $d$.

Theorem 4. Let $\alpha<1$ and $B$ be a bounded Borel set. If $\beta<1$

$$
P_{x}\left(X_{W_{B^{-}}} \in d y\right) \sim \tilde{g}(-x) \hat{\mu}_{B}(d y), \quad x \rightarrow \infty,
$$

and

$$
P_{x}\left(X_{T_{B}} \in d y\right) \sim \tilde{g}(-x) \mu_{B}(d y), \quad x \rightarrow \infty .
$$

If $\beta>-1$

$$
P_{x}\left(X_{W_{B^{-}}} \in d y\right) \sim \tilde{g}(-x) \hat{\mu}_{B}(d y), \quad x \rightarrow-\infty,
$$

and

$$
P_{x}\left(X_{T_{B}} \in d y\right) \sim \tilde{g}(-x) \mu_{B}(d y), \quad x \rightarrow-\infty .
$$

If $\beta=1, \gamma=[b \cos (\pi \alpha / 2)]^{1 /(1-\alpha)}$ and $d=\sup \{y: y \in B\}$

$$
P_{x}\left(X_{W_{B^{-}}} \in d y\right)=\frac{1}{b(1-\alpha)} e^{\gamma(y-x)} \hat{\mu}_{B}(d y), \quad x>d,
$$

and

$$
P_{x}\left(X_{T_{B}} \in d y\right)=e_{d}(d y) e^{\gamma(d-x)}, \quad x>d .
$$

One interesting consequence of Theorem 4 is that for $|\beta|<1$

$$
\begin{aligned}
\lim _{x \rightarrow \infty} P_{x}\left(X_{T_{B}} \in d y \mid T_{B}<\infty\right) & =\lim _{x \rightarrow-\infty} P_{x}\left(X_{T_{B}} \in d y \mid T_{B}<\infty\right) \\
& =\mu_{B}(d y) / C(B)
\end{aligned}
$$


and

$$
\begin{aligned}
\lim _{x \rightarrow \infty} P_{x}\left(X_{W_{B^{-}}} \in d y \mid T_{B}<\infty\right) & =\lim _{x \rightarrow-\infty} P_{x}\left(X_{W_{B^{-}}} \in d y \mid T_{B}<\infty\right) \\
& =\hat{\mu}_{B}(d y) / C(B) .
\end{aligned}
$$

The first relationship shows that the normalized capacitory measure can be viewed as the conditional first hitting distribution at $\infty$. The fact that left and right conditional limits are the same suggest that the process must jump over $B$ before hitting $B$ and in fact must jump from $-\infty$ to $\infty$ and vice versa before hitting $B$.

These relationships are false for processes with $\alpha<1$ and $\beta= \pm 1$. For $\beta=-1$ they fail to make sense since these processes can only move to the left. The verification that (1.7) fails to hold for processes with $\beta=1$ will be given in the section on proofs.

Theorem 5. Let $\alpha<1$. Then uniformly in $x$ on compacts as $t \rightarrow \infty$,

$$
p(t, x) t^{1 / \alpha} \rightarrow f(0)
$$

where

$$
\begin{aligned}
f(0) & =\frac{1}{\pi} \int_{0}^{\infty} e^{-\theta^{\prime \prime}} \cos \left(h \theta^{\alpha}\right) d \theta \\
& =\left[\frac{\Gamma(1 / \alpha)}{\alpha \pi}\right]\left[1+h^{2}\right]^{-1 / 2 \alpha} \cos \left(\frac{1}{\alpha} \tan ^{-1}(h)\right),
\end{aligned}
$$

and for $\beta=1, \gamma=(1-\alpha) \alpha^{\alpha /(1-\alpha)} \cos (\pi \alpha / 2)^{-1 /(1-\alpha)} b^{-\alpha / 1-\alpha}$,

$$
p(t, x) e^{\gamma t} \rightarrow \alpha^{1 / 2(1-\alpha)}(2 \pi(1-\alpha))^{-1 / 2} b^{-1+\alpha / 2(1-\alpha)} .
$$

Let $B$ be a bounded Borel set and let $|\beta|<1$. Then for any Borel set $A$,

$$
t^{1 / \alpha} P_{x}\left(X_{W_{B^{-}}} \in A, W_{B} \in d t\right) / d t \rightarrow f(0) \hat{\mu}_{B}(A)
$$

and

$$
t^{(1 / \alpha)-1} P_{x}\left(t<T_{B}<\infty, X_{T_{B}} \in A\right) \rightarrow \frac{\alpha f(0)}{1-\alpha} P_{x}\left(T_{B}=\infty\right) \mu_{B}(A) .
$$

Let $E_{B}(t, A)=\int P_{x}\left(T_{B} \leq t, X_{T_{B}} \in A\right) d x$. Then $E_{B}(t, A) \sim t \mu_{B}(A)$ and for $|\beta|<1$

$$
E_{B}(t, A)-t \mu_{B}(A) \uparrow \widehat{P}_{x}\left(T_{B}<\infty\right) P_{x}\left(X_{T_{B}} \in A\right) d x
$$

if $\alpha<1 / 2$. If $\alpha=1 / 2$

and if $1 / 2<\alpha<1$

$$
E_{B}(t, A)-t \mu_{B}(A) \sim C(B) \mu_{B}(A) \frac{\alpha}{1-\alpha} f(0) \ln t
$$

$$
E_{B}(t, A)-t \mu_{B}(A) \sim C(B) \mu_{B}(A)\left(\frac{\alpha^{2}}{1-\alpha}\right)\left(\frac{1}{2 \alpha-1}\right) t^{2-1 / \alpha}
$$

We now consider processes with $\alpha>1$. Here the drift substantially modifies the process compared to the drift free case. We first determine the asymptotic behavior of $g(x)$ as $x \rightarrow \pm \infty$. The asymptotic behavior as $x \rightarrow \pm 0$ is given by Theorems 13 and 15 . 
Theorem 6. Let $\alpha>1$ and let

$$
A_{j}(\alpha, \beta)=\frac{(-1)^{j+1} \Gamma(j \alpha+1)}{j ! \pi}\left(1+h^{2}\right)^{j / 2} \sin \left[j\left[\frac{\pi \alpha}{2}+\tan ^{-1}(h)\right]\right] \text {. }
$$

Then as $x \rightarrow \infty$

$$
g(x)=\sum_{j=1}^{n} A_{j}(j+1)^{-1}\left(\begin{array}{c}
\alpha j \\
j+1
\end{array}\right)^{-1} b^{-(j+1)} x^{j(1-\alpha)}+O\left(x^{(n+1)(1-\alpha)}\right) .
$$

If $\beta=-1$ and $\gamma=[b|\cos (\pi \alpha / 2)|]^{1 / \alpha-1}$ then

$$
g(x)=[(\alpha-1) b]^{-1} e^{-\gamma x}, \quad x>0 .
$$

For all $\beta$,

$$
g(0)=\frac{\alpha p}{(\alpha-1) b}
$$

where $p$ is given by (1.4). If $\beta=1, g(x)=1 / b$ for all $x \leq 0$ and is decreasing for $x>0$. For any $\beta$ and $x>0$,

$$
g(-x)=\frac{1}{b}+A_{1}(\alpha,-\beta)\left[\alpha(\alpha-1) b^{2}\right]^{-1} x^{1-\alpha}+\varepsilon(x),
$$

where $\varepsilon(x)=O\left(x^{2(1-\alpha) / \alpha}\right)$. Let $1<k<\infty$ and let $1 / k \leq y / x \leq k$. Then

$$
\lim _{x, y \rightarrow \infty}[g(x)-g(y)]\left[x^{1-\alpha}-y^{1-\alpha}\right]^{-1}=A_{1}(\alpha, \beta)\left[\alpha(\alpha-1) b^{2}\right]^{-1}
$$

and

(1.14b) $\lim _{x, y \rightarrow \infty}[g(-x)-g(-y)]\left[x^{1-\alpha}-y^{1-\alpha}\right]^{-1}=A_{1}(\alpha,-\beta)\left[\alpha(\alpha-1) b^{2}\right]^{-1}$.

The quantities $g(x)$ and $g(-x)$ are asymptotically strictly decreasing as $x \rightarrow \infty$ for all $\beta \neq 1$.

The probability of hitting $x$ starting from 0 is $h(x)=g(x) / g(0)$ $=[(\alpha-1) b) / \alpha p] g(x)$. If $\beta=1$, then $\alpha p=\alpha-1$ and $h(x)=1$ for all $x \leq 0$. If $\beta=-1$, then $\alpha p=1$ and $h(x)=e^{-\gamma x}, x>0$. For all $\beta$,

$$
\lim _{x \rightarrow-\infty} h(x)=\frac{\alpha-1}{\alpha p}
$$

and for $\beta>-1$

$$
h(x) \sim A_{1}\left[\alpha^{2} p b\right]^{-1} x^{1-\alpha}, \quad x \rightarrow \infty .
$$

Thus to first order the probability of hitting a point far to the left does not depend on $b$ while the probability of hitting a point far to the right is inversely proportional to $b$. This is in contrast to the processes with $\alpha<1$ where the probability of hitting points far from the origin is proportional to $b(|\beta|<1)$.

The asymptotic properties of the potential kernel given in Theorem 6 can be used to derive the asymptotic properties of the first and last hitting distributions for a bounded Borel set $B$. The arguments needed are the same as those used to establish Theorem 4 and will therefore be omitted. 
Theorem 7. Let $\alpha>1$ and let $B$ be a bounded Borel set. Then as $x \rightarrow \infty$

$$
P_{x}\left(X_{W_{B}-} \in d y\right) \rightarrow \frac{1}{b} \hat{\mu}_{B}(d y), \quad P_{x}\left(X_{T_{B}} \in d y\right) \rightarrow \frac{1}{b} \mu_{B}(d y) .
$$

If $\beta>-1$ then as $x \rightarrow-\infty$

$$
\begin{gathered}
|x|^{\alpha-1} P_{x}\left(X_{W_{B^{-}}} \in d y\right) \rightarrow A\left[\alpha(\alpha-1) b^{2}\right]^{-1} \hat{\mu}_{B}(d y), \\
|x|^{\alpha-1} P_{x}\left(X_{T_{B}} \in d y\right) \rightarrow A\left[\alpha(\alpha-1) b^{2}\right]^{-1} \mu_{B}(d y) .
\end{gathered}
$$

If $\beta=-1$ and $d=\inf \{x: x \in B\}$, then for $x<d$

$$
\begin{gathered}
P_{x}\left(X_{W_{B^{-}}} \in d y\right)=\frac{\alpha p}{(\alpha-1) b} e^{-\gamma(y-x)} \hat{\mu}_{B}(d y), \\
P_{x}\left(X_{T_{B}} \in d y\right)=e^{-\gamma(d-x)} e_{d}(d y) .
\end{gathered}
$$

Here $A$ is given by (1.11) and $\gamma$ is as in Theorem 6.

There are some interesting consequences of Theorem 7. Suppose $\beta>-1$. Then

$$
\begin{aligned}
\lim _{x \rightarrow \infty} P_{x}\left(X_{T_{B}} \in d y \mid T_{B}<\infty\right) & \left.=\lim _{x \rightarrow-\infty} P_{x}\left(X_{T_{B}} \in d y\right) \mid T_{B}<\infty\right) \\
& =\mu_{B}(d y) / C(B)
\end{aligned}
$$

and

$$
\begin{aligned}
\lim _{x \rightarrow \infty} P_{x}\left(X_{W_{B^{-}}} \in d y \mid T_{B}<\infty\right) & =\lim _{x \rightarrow-\infty} P_{x}\left(X_{W_{B^{-}}} \in d y \mid T_{B}<\infty\right) \\
& =\hat{\mu}_{B}(d y) / C(B) .
\end{aligned}
$$

The first of these relations shows (as for the $\alpha<1,|\beta|<1$ cases) that the normalized capacitory measure is the conditional hitting distribution at $\infty$. The fact that the left and right limits are the same has the same intuitive interpretation as for the $\alpha<1$ case. Note that if $\beta=1$ the process moves continuously to the left. Since $X_{t} \rightarrow-\infty$ with probability one it must be that for $x>\sup \{y: y \in B\}=d$ that $P_{x}\left(T_{B}<\infty\right)=1$ and the LHS of (1.15) is $e_{d}(d y)$, the unit mass at $d$. In this case the process starting from the far left must jump over $B$ before hitting $B$. Let $d^{\prime}=\inf \{y: y \in B\}$. Then for $\beta=1$, $P_{x}\left(T_{B}<\infty\right)=P_{x}\left(T_{d^{\prime}}<\infty\right)$.

Equation (1.15) is false in general for the processes with $\beta=-1$. This will be shown in the section having proofs.

If $\alpha<1$ the $\{t: X(t)=0\}$ is a finite set with probability one. If $\alpha>1$ this set is infinite and its size is measured by its Hausdorff dimension. Now for $\alpha>1, p(t, 0)=t^{-1 / \alpha} f\left(t^{-1 / \alpha} b\right) \sim t^{-1 / \alpha} f(0), t \rightarrow 0$. Using this fact and Theorem 4.1 of [1] it follows that the Hausdorff dimension of the $\{t: X(t)=0\}$ is $1-1 / \alpha$ just as for the drift free process.

Theorem 8. Suppose $\alpha>1$. If $\beta>-1$ and $A=A_{1}$ is given by (1.10) then uniformly in $x$ on compacts $p(t, x) t^{\alpha} \rightarrow A b^{-(\alpha+1)}, t \rightarrow \infty$. Also for any 
bounded Borel set $B$

$$
\begin{gathered}
t^{\alpha} P_{x}\left(X_{W_{B^{-}}} \in C, W_{B} \in d t\right) / d t \rightarrow A b^{-(\alpha+1)} \hat{\mu}_{B}(C), \\
t^{\alpha} P_{x}\left(X_{T_{B}} \in C, t<T_{B}<\infty\right) \rightarrow A b^{-(\alpha+1)} P_{x}\left(T_{B}=\infty\right) \mu_{B}(C) .
\end{gathered}
$$

Additionally if $E_{B}(t, C)=\int P_{x}\left(T_{B} \leq t, X_{T_{B}} \in C\right) d x$ than $E_{B}(t, C) \sim t \mu_{B}(C)$ and

$$
E_{B}(t, C)-t \mu_{B}(A) \sim C(B) \mu_{B}(C)[(2-\alpha)(\alpha-1)]^{-1} t^{2-\alpha} .
$$

If $\beta=-1$

$$
p(t, x) \sqrt{t} e^{\gamma t} \rightarrow A
$$

uniformly in $x$ on compacts where

$$
\gamma=(\alpha-1)|b / \alpha \cos (\pi \alpha / 2)|^{\alpha / \alpha-1}
$$

and

$$
A=[2 \pi(\alpha-1)]^{-1 / 2} \alpha^{-1 / 2(\alpha-1)} b^{-(-1+\alpha /(2(\alpha-1)))} .
$$

The processes with $\alpha>1$ go to $-\infty$ with probability one. Let $M=$ $\sup \left\{X_{t}: t \in(0, \infty)\right\}$. Then $M$ is finite with probability one. For a process starting at $X$ it is possible to explicitly compute the distribution of $M$ for Brownian motion and for the processes with $\beta=-1$. The result for Brownian motion is classical. The result for the processes with $\beta=-1$ follows from the fact that those processes move only continuously to the right. Thus for any $a>x, P_{x}(M \geq a)=P_{0}\left(T_{[a-x, \infty)}<\infty\right)=P_{0}\left(T_{a-x}<\infty\right)$. Hence by Theorem 6, $P_{x}(M>a)=e^{-\gamma(a-x)}$ where $\gamma$ is as in Theorem 6. Thus $M$ is exponentially distributed on $[x, \infty)$ with mean $\gamma^{-1}$.

It does not seem possible to explicitly compute the distribution of $M$ for the other processes with $\alpha>1$. However we can determine the asymptotic tail behavior of the distribution of $M$ for these processes. Since $P_{x}(M>a)=$ $P_{x}\left(T_{[a, \infty)}<\infty\right)$ the problem of finding the asymptotic behavior of $P_{x}(M>a)$, $a \rightarrow \infty$, is the same as finding the asymptotic hitting probability of $[a, \infty)$ as $a \rightarrow \infty$.

Theorem 9. Let $1<\alpha<2, \beta>-1$, and $A=A_{1}$ be given by (1.10). Then as $a \rightarrow \infty$

$$
P_{x}\left(T_{[a, \infty)}<\infty\right) a^{\alpha-1} \rightarrow A[\alpha(\alpha-1) b]^{-1} .
$$

A useful reformulation of (1.16) follows from the fact that

$$
P_{x}\left(T_{[a, \infty)}<\infty\right)=P_{0}\left(T_{[a-x, \infty)}<\infty\right)
$$

and the fact that $(a-x)^{\alpha-1} \sim-x^{\alpha-1}, x \rightarrow-\infty$. Thus

$$
\lim _{x \rightarrow-\infty} P_{x}\left(T_{[a, \infty)}<\infty\right)(-x)^{\alpha-1}=A[\alpha(\alpha-1) b]^{-1} .
$$

By Theorem 6

$$
\lim _{x \rightarrow-\infty} g(-x)(-x)^{\alpha-1}=A\left[\alpha(\alpha-1) b^{2}\right]^{-1} .
$$


Hence (1.17) is equivalent to

$$
P_{x}\left(T_{[a, \infty)}<\infty\right) \sim g(-x) b^{-1}, \quad x \rightarrow-\infty .
$$

Let $B$ be a bounded Borel subset of $[a, \infty)$. Then

$$
P_{x}\left(X_{T_{B}} \in d y \mid T_{[a, \infty)}<\infty\right)=P_{x}\left(T_{B} \in d y\right) / P_{x}\left[T_{[a, \infty)}<\infty\right) .
$$

Using Theorem 7 and (1.17) we find for $1<\alpha<2$, and $\beta>-1$

$$
\lim _{x \rightarrow-\infty} P_{x}\left(X_{T_{B}} \in d y \mid T_{[a, \infty)}<\infty\right)=\mu_{B}(d y) / b
$$

and thus by Theorem 7 the following curious relationship holds.

Corollary 1. Let $1<\alpha<2$ and $\beta>-1$. Then for $B$ a bounded Borel subset of $[a, \infty)$

$$
\lim _{x \rightarrow-\infty} P_{x}\left(X_{T_{B}} \in d y \mid T_{[a, \infty)}<\infty\right)=\lim _{x \rightarrow \infty} P_{x}\left(X_{T_{B}} \in d y\right)=\frac{\mu_{B}(d y)}{b} .
$$

In particular (1.19) shows

$$
\lim _{x \rightarrow-\infty} P_{x}\left(T_{B}<\infty \mid T_{[a, \infty)}<\infty\right)=\lim _{x \rightarrow \infty} P_{x}\left(T_{B}<\infty\right)=C(B) / b .
$$

Taking $B=\{d\}(1.20)$ shows that for $d \in[a, \infty)$

$$
\lim _{x \rightarrow-\infty} P_{x}\left(T_{d}<\infty \mid T_{[a, \infty)}<\infty\right)=\lim _{x \rightarrow \infty} P_{x}\left(T_{d}<\infty\right)=\frac{\alpha-1}{\alpha p} .
$$

It is interesting that in (1.21) the limiting probabilities do not depend on $b$.

For processes with $\beta=-1,1<\alpha<2$, these relationships can fail. For example in (1.21) take $d=a$. Since processes with $\beta=-1$ move only continuously to the right the LHS of (1.21) is 1 . However for these processes $\alpha p=1$ so the RHS is $\alpha-1$. Thus (1.21) fails for processes with $\beta=-1$.

Recall that the occupation time of a set $B$ is the Lebesgue measure of the set $\left\{t: X_{t} \in B\right\}$. Let $a>0$ and let $N(a)$ be the occupation time of $[0, a]$ for a process starting from 0 and let $N(-a)$ be the occupation time of $[-a, 0]$ for a process starting from 0 . Let $\tilde{N}(a)$ and $\tilde{N}(-a)$ be these quantities for the corresponding drift free process when $\alpha<1$. The scaling relationship for strictly stable processes shows that

$$
\tilde{N}(a) \stackrel{D}{=} a^{\alpha} \tilde{N}(1) \text { and } \tilde{N}(-a) \stackrel{D}{=} a^{\alpha} \tilde{N}(-1) .
$$

Theorem 10. Assume $\alpha<1$. Then as $a \rightarrow \infty, N(a) a^{-\alpha} \stackrel{D}{\rightarrow} \widetilde{N}(1), N(-a) a^{-\alpha}$ $\stackrel{D}{\rightarrow} \tilde{N}(-1)$ and $a^{-\alpha}[N(a)+N(-a)] \stackrel{D}{\rightarrow} \widetilde{N}(1)+\widetilde{N}(-1)$.

The distribution of $\tilde{N}(-1)$ for a process with parameter $(\alpha, \beta)$ is the same as the distribution of $\tilde{N}(1)$ for the process with parameter $(\alpha,-\beta)$. If $\beta=-1$, $\widetilde{N}(1)=0$ and if $\beta=+1, \widetilde{N}(1) / \Gamma(\alpha)$ is the Mittag-Leffler distribution of index $\alpha$. Recall this is the distribution that is uniquely determined by its moments which are

$$
r ![\Gamma(\alpha r+1)]^{-1} .
$$


For $|\beta|<1$ the distributions of $\tilde{N}(1)$ and $\tilde{N}(1)+\tilde{N}(-1)$ are also uniquely determined by their moments but do not coincide with those of any known distribution. The moments are given by

$$
\mathrm{E} \tilde{N}(1)^{r}=r ! \int_{0}^{1} \cdots \int_{0}^{1} \tilde{g}\left(x_{1}\right) \cdots \tilde{g}\left(x_{2}-x_{1}\right) \cdots \tilde{g}\left(x_{r}-x_{r-1}\right) d x_{1} \cdots d x_{r}
$$

and

$$
\mathrm{E}[\tilde{N}(1)+\tilde{N}(-1)]^{r}=r ! \int_{-1}^{1} \cdots \int_{-1}^{1} \tilde{g}\left(x_{1}\right) \cdots \tilde{g}\left(x_{r}-x_{r-1}\right) d x_{1} \cdots d x_{r} .
$$

The first two moments of $N(1)$ can be explicitly computed but it does not seem possible to give a formula for the moments $\geq 3$. Let

$$
K_{+}=\left(1+h^{2}\right)^{-1}\left(\frac{\Gamma(1-\alpha)}{\pi}\right) \sin \left(\frac{\alpha \pi}{2}\right)(1+\beta)
$$

and

$$
K_{-}=\left(1+h^{2}\right)^{-1}\left[\frac{\Gamma(1-\alpha)}{\pi}\right]^{-1} \sin \left(\frac{\alpha \pi}{2}\right)(1-\beta)
$$

Then

$$
\mathrm{E} \tilde{N}(1)=K_{+} / \alpha, \quad \mathrm{E} \tilde{N}(1)^{2}=2 K_{+}\left[\frac{K_{-}}{2 \alpha^{2}}+K_{+} \frac{\Gamma(\alpha)^{2}}{\Gamma \alpha+1)}\right] .
$$

The range of the process $X_{t}$ is the random set $\left\{x: X_{t}=x\right.$ for some $\left.t\right\}$. For $a>0$ let $R(a)$ be the Lebesgue measure of the range intersected with $[0, a]$ and let $R(-a)$ be the Lebesgue measure of the range intersected with $[-a, 0]$. Using Theorem 10 and Theorem 1 of [8] we obtain the following.

Theorem 11. Let $\alpha<1$. Then $R(a) / C a^{\alpha} \stackrel{D}{\rightarrow} \widetilde{N}(1), R(-a) / C a^{\alpha} \stackrel{D}{\rightarrow} \widetilde{N}(-1)$, and $[R(a)+R(-a)]\left[C a^{\alpha}\right]^{-1} \stackrel{D}{\rightarrow} \tilde{N}(1)+\widetilde{N}(-1)$.

For $\alpha>1$ things are considerably different. Theorem 4 of [8] shows that the following holds.

Theorem 12. Let $\alpha>1$. Then as $a \rightarrow \infty$, (i) $N(a) \uparrow N(\infty)$, the occupation time of $[0, \infty)$, and $R(a) \uparrow R(\infty)$, the Lebesgue measure of the range intersected with $(0, \infty)$.

(ii) $N(-a) / a \rightarrow 1 / b$ and $R(-a) / a \rightarrow C / b=(\alpha-1) / \alpha p$ with probability one.

We will now establish the asymptotic behavior of $g(x)$ for $x$ near 0 .

Theorem 13. Suppose $\alpha>1$. Let

$$
I(\alpha, \beta)=\Gamma(2-\alpha)[(\alpha-1) \pi]^{-1}\left(1+h^{2}\right)^{-1 / 2} \cos \left[\frac{\pi(\alpha-1)}{2}+\tan ^{-1}(h)\right] .
$$

Then as $x \downarrow 0$

$$
g(x)=g(0)-I(\alpha, \beta) x^{\alpha-1}+\varepsilon(x)
$$


and

$$
g(-x)=g(0)-I(\alpha,-\beta) x^{\alpha-1}+\varepsilon(x)
$$

where $\varepsilon(x)=O\left(x^{2(\alpha-1)}\right)$ if $\alpha<3 / 2$ and $\varepsilon(x)=O(x)$ if $3 / 2 \leq \alpha<2$.

Theorem 14. Suppose $\alpha<1$. Let $A=A_{1}$ be given by (1.10) and let $A_{-}(\alpha, \beta)=$ $A(\alpha,-\beta)$. Then as $x \downarrow 0$

$$
\begin{gathered}
g(x)=g(0)-A\left[\alpha(1-\alpha) b^{2}\right]^{-1} x^{1-\alpha}+O\left(x^{2(1-\alpha)}\right), \\
g(-x)=g(0-)-A_{-}\left[\alpha(1-\alpha) b^{2}\right]^{-1} x^{1-\alpha}+\varepsilon(x)
\end{gathered}
$$

where $\varepsilon(x)=x^{2(1-\alpha)}$ if $\alpha<1 / 2$ and $\varepsilon(x)=x^{(1-\alpha) / \alpha}$ if $\alpha \geq 1 / 2$.

We can also obtain asymptotic estimates of differences $g(y)-g(x)$ as $x, y \rightarrow$ 0 and $1 / k \leq|y / x| \leq k, 1<k<\infty$ and $x$ and $y$ have the same sign. The arguments are very similar to those used to establish the corresponding results for $x, y \rightarrow \infty$ in Theorems 3 and 6 and those used to establish Theorems 13 and 14. We will thus omit the proofs.

Theorem 15. Assume $x>0,1<k<0$, and $\frac{1}{k} x \leq y \leq k x$. Suppose $\alpha>1$. Then for $A$ as in Theorem 14,

$$
\begin{gathered}
g(y)-g(x) \sim I(\alpha, \beta)\left[y^{\alpha-1}=x^{\alpha-1}\right], \quad x, y \rightarrow 0, \\
g(-y)-g(-x) \sim I(\alpha,-\beta)\left[y^{\alpha-1}-x^{\alpha-1}\right], \quad x, y \rightarrow 0 .
\end{gathered}
$$

Assume $\alpha<1$. Then

$$
g(y)-g(x) \sim A(\alpha, \beta)\left[\alpha(1-\alpha) b^{2}\right]^{-1}\left[y^{1-\alpha}-x^{1-\alpha}\right], \quad x, y \rightarrow 0,
$$

and

$$
g(-y)-g(-x) \sim-A(\alpha,-\beta)\left[\alpha(1-\alpha) b^{2}\right]^{-1}\left[y^{1-\alpha}-x^{1-\alpha}\right], \quad x, y \rightarrow 0 .
$$

It follows from Theorem 15 that $g(x)$ is asymptotically strictly monotone for $x$ near 0 .

We now turn our attention to the Cauchy processes having drift $b$. We must here distinguish the processes with $\beta=0$ from those with $\beta \neq 0$.

Suppose $\beta \neq 0$. Examination of the characteristic function shows that $X_{t}=$ $c \widetilde{X}_{r t}$ for appropriate choice of $c$ and $r$. Consequently the results for these processes follow from the known facts about the asymmetric Cauchy processes without drift.

If $\beta=0$ one can easily explicitly compute the density of $X_{t}$. The result is

$$
P(t, x)=\frac{t}{\pi\left[t^{2}+(x+b t)^{2}\right]} .
$$

General theory shows that these processes are recurrent and that points are polar sets. The recurrent potential kernel is

$$
a(x)=\int_{0}^{\infty}[p(t, 1)-p(t, x)] d t
$$


This can be explicitly evaluated. The result is

$$
a(x)=\frac{1}{\pi\left(1+b^{2}\right)}\left[\ln |x|+\tan ^{-1}(b)-\tan ^{-1}(x b)\right]
$$

The explicit form of the kernel $a(x)$ and the fact that $p(t, x) \sim \frac{1}{t}\left(1+b^{2}\right)^{-1}$ enable one to easily apply the general theory in [6] to obtain explicit asymptotic formulas analogous to those in the above theorems. Since this amounts to just plugging in to the formulas in [6] we omit these details.

In [7] it was shown that the potential kernel $g(x)$ for an asymmetric Cauchy process satisfied the relationship

$$
g(x) \sim[1+\beta \operatorname{sgn}(x)] \frac{\pi}{4 \beta^{2}} \frac{1}{\ln |x|}, \quad x \rightarrow \pm \infty .
$$

Also the analogs of Theorems 4 and 5 were established for these processes in [7]. We will discuss here the analog of Theorem 16 for asymmetric Cauchy processes.

Theorem 16. Let $a>0$ and let $N(a)$ be the occupation time of $[0, a]$ by an asymmetric Cauchy process and $N(-a)$ the occupation time of $[-a, 0]$ by this process. Let $Z$ be a geometrically distributed random variable having parameter $\rho$ [i.e. $P(Z=j)=(1-\rho) \rho^{j}, j=0,1, \ldots$ ]. Then as $a \rightarrow \infty, N(a) \frac{\ln a}{a}$ converges in distribution to $N^{+}, N(-a) \frac{\ln a}{a}$ converges in distribution to $N^{-}$, and $N(a)+N(-a)$ converges in distribution to $N$. Assume $\beta>0$ and let $\rho=(1-\beta) /(1+\beta)$. Then $N^{+} \underline{\underline{D}}(\pi / 2 \beta)(Z+1), N^{-} \underline{\underline{D}}(\pi / 2 \beta) Z$ and $N \stackrel{D}{=}$ $(\pi / 2 \beta)(2 Z+1)$.

Remark. The corresponding results about the asymptotic distributions of $N(a)$, $N(-a)$ and $N(a)+N(-a)$ for processes with $\beta<0$ follows from those in Theorem 16 since the process $-X(t)$ is the same as a process $X(t)$ with $\beta$ replaced with $-\beta$. It is of course striking that the limiting distributions are discrete and, except for $|\beta|=1$, nondegenerate. Such strange limiting distributions were first found by Pruitt and Taylor [9] in their investigation of $R(a)$ and $R(-a)$. They showed that $R(a) \ln a / a \stackrel{D}{\rightarrow} R^{+}, R(-a) \ln a / a \stackrel{D}{\rightarrow} R^{-}$, and $[R(a)+R(-a)] \ln a / a \stackrel{D}{\rightarrow} R$ where $R^{+} \stackrel{D}{=} Z+1, R^{-} \stackrel{D}{=} Z, R \stackrel{D}{=}(2 Z+1)$. Since $2 \beta / \pi=C$ we see that $R^{+} \stackrel{D}{=} C N^{+}, R^{-} \stackrel{D}{=} C N^{-}$, and $R \stackrel{D}{=} C N$. Theorem 1 of [8] explains the connection between the limiting distributions of $N(a)$ and $R(a)$.

\section{Proofs}

We will need several well-known facts about the density $f(x)$. These may all be found in [5] or are simple consequences of the facts in [5]. The asymptotic formulas given here are taken from Skorohod [10] since those in [5] are for a stable process having a scale factor $\neq 1$.

The density $f$ has bounded continuous derivatives of all orders. 
Proposition 2.1. Assume $\alpha \neq 1,2$. Then

$$
f(x) \sim A_{+} x^{-(\alpha+1)}, \quad x \rightarrow \infty,
$$

where

$$
A_{+}=\frac{\Gamma(\alpha+1)}{\pi}\left[1+\beta^{2} \tan \left(\frac{\pi \alpha}{2}\right)\right]^{1 / 2} \sin \left[\frac{\pi \alpha}{2}+\tan ^{-1}(h)\right]
$$

and $f(x) \sim A_{-}|x|^{-(\alpha+1)}, x \rightarrow-\infty$, where

$$
A_{-}=\frac{\Gamma(\alpha+1)}{2}\left(1+\beta^{2} \tan \left(\frac{\pi \alpha}{2}\right)\right)^{1 / 2} \sin \left(\frac{\pi \alpha}{2}-\tan ^{-1}(h)\right) .
$$

Also for $n=1,2, \ldots$,

$$
f^{(n)}(x) \sim(-1)^{n}(\alpha+1) \cdots(\alpha+n) A_{+} x^{-(\alpha+n+1)}, \quad x \rightarrow \infty,
$$

and

$$
f^{(n)}(-x) \sim(-1)^{n}(\alpha+1) \cdots(\alpha+n) A_{-} x^{-(\alpha+n+1)}, \quad x \rightarrow \infty .
$$

Additionally

$$
\sup _{x}\left|f^{(n)}(x)\right||x|^{\alpha+n+1}=k_{n}<\infty .
$$

Proposition 2.2. Let $\alpha<1$ and $\beta=1$. Let

$$
\lambda=\frac{\alpha}{1-\alpha}, \quad A=\alpha^{1 / 2(1-\alpha)}\left[\cos \left(\frac{\pi \alpha}{2}\right)\right]^{-1 / 2(1-\alpha)}[2 \pi(1-\alpha)]^{-1 / 2},
$$

and $B=(1-\alpha) \alpha^{\lambda}[\cos (\pi \alpha / 2)]^{-1 /(1-\alpha)}$. Then

$$
f(x) \sim A x^{-(1+\lambda / 2)} e^{-B x^{-\lambda}}, \quad x \downarrow 0 .
$$

Proposition 2.3. Let $1<\alpha<2$ and $\beta=-1$. Let

$$
\lambda=\frac{\alpha}{\alpha-1}, \quad A=\alpha^{-1 /(2(1-\alpha))}\left|\cos \left(\frac{\pi}{2} \alpha\right)\right|^{1 /(2(\alpha-1))}[2 \pi(\alpha-1)]^{1 / 2},
$$

and

$$
B=(\alpha-1) \alpha^{-\lambda}\left|\cos \left(\frac{\pi}{2} \alpha\right)\right|^{1 /(\alpha-1)} .
$$

Then

$$
f(x) \sim A x^{-1+\lambda / 2} e^{-B x^{\lambda}}, \quad x \rightarrow \infty .
$$

Proposition 2.4. Let $\alpha \neq 1,2$, and let

$$
A_{j}(\alpha, \beta)=(-1)^{j+1} \frac{\Gamma(j \alpha+1)}{j ! \pi}\left(1+h^{2}\right)^{j / 2} \sin \left[j\left[\frac{\pi \alpha}{2}+\tan ^{-1}(h)\right]\right] .
$$

Then for any $n$ as $x \rightarrow \infty$

$$
f(x)=\sum_{j=1}^{n} A_{j} x^{-(j \alpha+1)}+O\left(x^{-(\alpha(n+1)+1)}\right),
$$


and

$$
f(-x)=\sum_{j=1}^{n} A_{j}(\alpha,-\beta)(x)^{-(j \alpha+1)}+O\left((x)^{-(\alpha(n+1)+1)}\right) .
$$

These formulas can be differentiated any numbers of times. There are finite constants $K_{1}$ and $K_{2}$ such that for all $x$

$$
\left.\left.\left|f(x)-\sum_{j=1}^{n} A_{j}\right| x\right|^{-(\alpha+1)}\left|\leq K_{1}\right| x\right|^{-[(n+1) \alpha+1]}
$$

and

$$
\left.\left.\left|f^{\prime}(x)+\sum_{j=1}^{n}(\alpha j+1) A_{j}\right| x\right|^{-(\alpha j+2)}\left|\leq K_{2}\right| x\right|^{-[(n+1) \alpha+2]} .
$$

Proof. For the expansions of $f$ see [8]. For the expansion on the derivative see [5]. The bounds on $f$ and $f^{\prime}$ follow from these expansions and the fact that $f$ and $f^{\prime}$ are bounded continuous functions.

It is also known (see [2]) that

$$
p=\int_{0}^{\infty} f(x) d x=\frac{1}{2}+\tan ^{-1}(h) / \pi \alpha .
$$

Proof of Theorem 1. Now

$$
g(0)=\int_{0}^{\infty} t^{-1 / \alpha} f\left(t^{1-1 / \alpha} b\right) d t=\frac{\alpha p}{(1-\alpha) b} .
$$

The results in Theorem 1 now follow at once from (2.9) and the following fact.

$$
g(0-)-g(0)=\frac{1}{b} \text {. }
$$

This last fact, as pointed out by the referee, is actually valid whenever $X_{t}$ is a transient levy process having no Gaussian component, drift $-b$, and Levy measure $M$ such that $\int_{|x| \leq 1} M(d x)|x|<\infty$. A statement of this fact, at least for $g$ replaced by the $\lambda$-potential kernel, can be found on p. 289 of [4]. However, no proof is given and as the proof is not all together routine I shall supply a proof in the appendix to this paper.

Proof of Theorem 2. The process can only move to the left continuously. Let $h(x)=P_{0}\left(T_{x}<\infty\right)$. The spatial homogeneity and the strong Markov property show

$$
h(-(x+y))=h(-x) h(-y), \quad x, y>0 .
$$

Since $0 \leq h(x) \leq 1, h(-x)$ is decreasing. Thus there is a $\gamma$ such that $h(-x)=$ $e^{-\gamma x}$. But then

$$
e^{-\gamma x}=g(-x) C, \quad x>0 .
$$

To find $\gamma$ we will make use of Proposition 2.2. Since

$$
g(-x)=\int_{x / b}^{\infty} t^{-1 / \alpha} f\left(t^{-1 / \alpha}(-x+b t)\right) d t
$$


it follows from Proposition 2.2 that as $x \rightarrow \infty$,

$$
\begin{aligned}
g(-x) & \sim A \int_{x / b}^{\infty} t^{-1 / \alpha}\left[t^{-1 / \alpha}(-x+b t)\right]^{-(1+\lambda / 2)} e^{-B\left[t^{-(1+\alpha)}(-x+b t)\right]^{-\lambda}} d t \\
& =\int_{1}^{\infty} A\left(\frac{x}{b}\right)^{1 / 2} s^{\lambda / 2 \alpha} b^{-(1+\lambda / 2)}(s-1)^{-(1+\lambda / 2)} e^{-B x b^{-(1+\lambda) s^{\lambda / \alpha}(s-1)^{-\lambda}} d s .}
\end{aligned}
$$

Let $\phi(s)=s^{\lambda / \alpha}(s-1)^{-\lambda}$. Observe that $\phi$ has a unique minimum at $s_{0}=$ $1 /(1-\alpha)$,

$$
\phi\left(s_{0}\right)=(1-\alpha)^{-\lambda / \alpha}(\alpha /(1-\alpha))^{-\lambda} \text { and } \phi^{\prime \prime}\left(s_{0}\right)=\phi\left(s_{0}\right)(1-\alpha)^{2} / \alpha .
$$

To evaluate the asymptotic behavior of the integral on the extreme right of (2.11) decompose that integral into three terms $I_{1}=\int_{1}^{(1+a) s_{0}}, I_{2}=\int_{(1-a) s_{0}}^{(1+a) s_{0}}$, and $I_{3}=\int_{(1+a) s_{0}}^{\infty}$. We first consider $I_{2}$. Let $\varepsilon>0$ be specified and be such that $\phi^{\prime \prime}\left(s_{0}\right)-\varepsilon>0$, choose $a>0$ such that $\left|\phi^{\prime \prime}(s)-\phi^{\prime \prime}\left(s_{0}\right)\right|<\varepsilon$ for $\left|s-s_{0}\right|<a$, and also $\left|s^{\lambda / 2 \alpha}(s-1)^{-(1+\lambda / 2)}-s_{0}^{\lambda / 2 \alpha}\left(s_{0}-1\right)^{-(1+\lambda / 2)}\right|<\varepsilon$ for $\left|s-s_{0}\right|<a$. Let

$$
\begin{gathered}
d=s_{0}^{\lambda / 2 a}\left(s_{0}-1\right)^{-(1+\lambda / 2)}=\left(\frac{1}{1-\alpha}\right)^{\lambda / 2 \alpha}\left(\frac{\alpha}{1-\alpha}\right)^{-(1+\lambda / 2)}, \\
\gamma^{\prime}=B b^{-(1+\lambda)} \phi\left(s_{0}\right) \text {, and } B^{\prime}=B b^{-(1+\lambda)} \text {. Then } \\
e^{\gamma^{\prime} x} I_{2} \leq \int_{(1-a) s_{0}}^{(1+a) s_{0}} A\left(\frac{x}{b}\right)^{1 / 2}[d+\varepsilon] e^{-B^{\prime} / 2\left[\phi^{\prime \prime}\left(s_{0}\right)-\varepsilon\right]\left(s-s_{0}\right)^{2}} d s .
\end{gathered}
$$

The change of variable $x B^{\prime} / 2\left[\phi^{\prime \prime}\left(s_{0}\right)-\varepsilon\right]\left(s-s_{0}\right)^{2}=z$ shows

$$
e^{\gamma^{\prime} x} I_{2} \leq \int_{0}^{x B^{\prime} / 2\left[\phi^{\prime \prime}\left(s_{0}\right)-\varepsilon\right]\left(a s_{0}\right)^{2}} A b^{-1 / 2}[d+\varepsilon] e^{-z} z^{-1 / 2}\left[\frac{B^{\prime}}{2}\left[\phi^{\prime \prime}\left(s_{0}\right)-\varepsilon\right]\right]^{-1 / 2} d z
$$

Thus

$$
\varlimsup_{x \rightarrow \infty} e^{\gamma^{\prime} x} I_{2} \leq \sqrt{2 \pi} A b^{-1 / 2}[d+\varepsilon]\left[B\left(\phi^{\prime \prime}\left(s_{0}\right)-\varepsilon\right)\right]^{-1 / 2} .
$$

An analogous computation shows

$$
\varliminf_{x \rightarrow \infty} e^{\gamma^{\prime} x} I_{2} \geq \sqrt{2 \pi} A b^{-1 / 2}[d-\varepsilon]\left[B^{\prime}\left(\phi^{\prime \prime}\left(s_{0}\right)+\varepsilon\right)\right]^{-1 / 2} .
$$

We now consider $I_{1}$. Observe that

$$
I_{1}=\int_{1}^{(1-a) s_{0}} A\left(\frac{x}{b}\right)^{1 / 2} \sqrt{\phi(s)}(s-1)^{-1} e^{-B^{\prime} x \phi(s)} d s .
$$

Thus

$$
e^{\gamma^{\prime} x} I_{1}=\int_{1}^{(1-a) s_{0}} A\left(\frac{x}{b}\right)^{1 / 2} \sqrt{\phi(s)}(s-1)^{-1} e^{-B^{\prime} x\left(\phi(s)-\phi\left(s_{0}\right)\right)} d s .
$$


Observe that $\phi^{\prime}(s)=\lambda \phi(s)(s(1-\alpha)-1)[(\alpha s)(s-1)]^{-1}$. The change of variable $u=\phi(s)$ in the above integral yields

$$
e^{\gamma^{\prime} x} I_{1}=\int_{\phi\left((1-a) s_{0}\right)}^{\infty} A\left(\frac{x}{b}\right)^{1 / 2} u^{1 / 2} e^{-B^{\prime} x\left(u-\phi\left(s_{0}\right)\right)} \alpha s[1-(1-\alpha) s]^{-1} d u .
$$

Now for all $s, 1 \leq s<\infty, \alpha \leq \alpha s(1-(1-\alpha) s)^{-1} \leq \alpha /(1-\alpha)$. Thus,

$$
e^{\gamma^{\prime} x} I_{1} \leq \frac{\alpha}{1-\alpha} \int_{\phi\left((1-a) s_{0}\right)}^{\infty} A\left(\frac{x}{b}\right)^{1 / 2} u^{1 / 2} e^{-B x\left(u-\phi\left(s_{0}\right)\right)} d u
$$

Hence $\lim _{x \rightarrow \infty} e^{\gamma^{\prime} x} I_{1}=0$. An analogous computation shows $\lim _{x \rightarrow \infty} e^{\gamma^{\prime} x} I_{3}=$ 0 . Using the above facts and (2.12) and (2.13) we now find

$$
\varlimsup_{x \rightarrow \infty} e^{\gamma^{\prime} x} g(-x) \leq \sqrt{2 \pi} A b^{-1 / 2}[d+\varepsilon]\left[B\left(\phi^{\prime \prime}\left(s_{0}\right)-\varepsilon\right)\right]^{-1 / 2} .
$$

Letting $\varepsilon \downarrow 0$ we find

$$
\varlimsup_{x \rightarrow \infty} e^{\gamma^{\prime} x} g(-x) \leq \sqrt{2 \pi} A b^{-1 / 2} d\left[B \phi^{\prime \prime}\left(s_{0}\right)\right]^{-1 / 2}=[(1-\alpha) b]^{-1} .
$$

Similarly

$$
\varliminf_{x \rightarrow \infty} e^{\gamma^{\prime} x} g(-x) \geq[(1-\alpha) b]^{-1}
$$

Thus

$$
\lim _{x \rightarrow \infty} e^{\gamma^{\prime} x} g(-x)=[(1-\alpha) b]^{-1}=1 / C .
$$

This shows that $\gamma=\gamma^{\prime}$. Thus $\gamma=[b \cos (\pi \alpha / 2)]^{-1 /(1-\alpha)}$.

Proof of Theorem 3. For $x>0$,

$$
\begin{aligned}
g(x) & =\int_{0}^{\infty} t^{-1 / \alpha} f\left(t^{-1 / \alpha} x+b t^{1-1 / \alpha}\right) d t \\
& =\left[\alpha \int_{0}^{\infty} f\left(u+b\left(\frac{u}{x}\right)^{1-\alpha}\right) u^{-\alpha} d u\right] x^{\alpha-1}
\end{aligned}
$$

By Taylor's theorem for any $n$

$$
g(x)=\sum_{j=0}^{n} \gamma_{j}^{+} b^{j} x^{(j+1)(\alpha-1)}+x^{(n+2)(\alpha-1)} R_{n}(x) b^{n+1}
$$

where for some $\xi, 0 \leq \xi \leq b(u / x)^{1-\alpha}$,

$$
R_{n}(x)=\frac{\alpha}{(n+1) !} \int_{0}^{\infty} f^{(n+1)}(u+\xi) u^{n+1-(n+2) \alpha} d u
$$

and

$$
\gamma_{j}^{+}=\frac{\alpha}{j !} \int_{0}^{\infty} f^{(j)}(u) u^{j-(j+1) \alpha} d u
$$

Now $u^{j-(j+1) \alpha}$ is integrable on $[0, A]$ for any $A<\infty$. Since $f^{(j)}$ is bounded, dominated convergence shows

$$
\lim _{A \uparrow \infty} \lim _{x \rightarrow \infty} \alpha \int_{0}^{A} \frac{f^{(n+1)}(u+\xi) u^{n+1-(n+2) \alpha} d u}{(n+1) !}=\gamma_{n+1}^{+}
$$


By Proposition 2.1 for $u>A$,

$$
\left|f^{(n+1)}(u+\xi)\right| u^{n+1-(n+2) \alpha} \leq k_{n+1} u^{-1-(n+3) \alpha} .
$$

Thus as $A \rightarrow \infty$,

$$
\int_{A}^{\infty} f^{(n+1)}(u+\xi) u^{n+1-(n+2) \alpha} d u \rightarrow 0
$$

Hence

$$
\lim _{x \rightarrow \infty}\left[g(x)-\sum_{j=0}^{n} \gamma_{j}^{+} b^{j} x^{(j+1)(\alpha-1)}\right] x^{(n+2)(1-\alpha)}=\gamma_{n+1}^{+} .
$$

An analogous argument shows

$$
\begin{aligned}
& \varlimsup_{x \rightarrow \infty}\left|\left[g(-x)-\sum_{j=0}^{n} \gamma_{j}^{-} b^{j} x^{(j+1)(\alpha-1)}\right]-\gamma_{n+1}^{-}\right| \\
& \quad \leq \lim _{A} \varlimsup_{x \rightarrow \infty} \int_{A}^{\infty}\left|f^{(n+1)}(-u+\xi) u^{n+1-(n+2) \alpha}\right| d u .
\end{aligned}
$$

Now for $x \geq 1$ and $u \geq(1 / 2 b)^{1 / \alpha}$ Proposition 2.1 shows

$$
\begin{aligned}
\left|f^{(n+1)}(-u+\xi)\right| & \leq k_{n+1} u^{-(n+2+\alpha)}|-1+\xi / u|^{-(n+2+\alpha)} \\
& \leq k_{n+1}\left(\frac{1}{2}\right)^{-(n+2+\alpha)} u^{-(n+2+\alpha)}
\end{aligned}
$$

Thus

$$
\lim _{A} \varlimsup_{x \rightarrow \infty} \int_{A}^{\infty}\left|f^{(n+1)}(-u+\xi) u^{n+1-(n+2) \alpha}\right| d u=0 .
$$

We now determine the coefficients $\gamma_{n}^{+}(\alpha, \beta)$ and $\gamma_{n}^{-}(\alpha, \beta)$. Since this is done by rather routine computation we will only outline the necessary steps. Let $f(x, \alpha, \beta)$ be the stable density with exponent $\alpha$ and asymmetry parameter $\beta$. Then $f(-x, \alpha, \beta)=f(x, \alpha,-\beta)$. Thus $\gamma_{n}^{-}(\alpha, \beta)=\gamma_{n}^{+}(\alpha,-\beta)$. Now

$$
\gamma_{n}^{+}(\alpha, \beta)=\frac{\alpha}{n !} \int_{0}^{\infty} f^{(n)}(u) u^{n-(n+1) \alpha} d u .
$$

Let

$$
\begin{aligned}
& E_{n}=\sin \left(\frac{\pi}{2}(n+1) \alpha\right), \quad F_{n}=\cos \left(\frac{\pi}{2}(n+1) \alpha\right), \\
& C_{n}=n !\left(1+h^{2}\right)^{-(n+1) / 2} \cos \left((n+1) \tan ^{-1}(h)\right), \\
& D_{n}=n !\left(1+h^{2}\right)^{-(n+1) / 2} \sin \left((n+1) \tan ^{-1}(h)\right) .
\end{aligned}
$$

To evaluate $I_{n}=\int_{0}^{\infty} f^{(n)}(u) u^{n-(n+1) \alpha} d u$ we need to know where $\alpha$ is. Suppose $(n-j) /(n+j)<\alpha<(n-j+1) /(n+1), j=0, \ldots, n$. Integrating by parts $j$ times shows

$$
I_{n}=\frac{(-1)^{j} \Gamma((n+1)(1-\alpha))}{\Gamma((n+1)(1-\alpha)-j)} \int_{0}^{\infty} f^{(n-j)}(u) u^{n-j-(n+1) \alpha} d u
$$


Using the Fourier inversion formula we find

$$
f^{(n-j)}(u)=\frac{1}{\pi} \int_{0}^{\infty} e^{-\theta^{n}} \psi\left(h \theta^{\alpha}-u \theta\right) \theta^{n-j} d \theta
$$

where $\psi(x)=\cos x$ if $n-j=4 r, \psi(x)=\sin (x)$ if $n-j=4 r+1, \psi(x)=$ $-\cos (x)$ if $n-j=4 r+2$, and $\psi(x)=-\sin (x)$ if $n-j=4 r+3$. Then

$$
\begin{aligned}
J_{n, j} & =\int_{0}^{\infty} f^{(n-j)}(u) u^{n-j-(n+1) \alpha} d u \\
& =\frac{1}{\pi} \int_{0}^{\infty} u^{n-j-(n+1) \alpha} d u \int_{0}^{\infty} \psi\left(h \theta^{\alpha}-u \theta\right) \theta^{n-j} d \theta .
\end{aligned}
$$

Interchanging the order of integration and making a change of variable we find

$$
J_{n, j}=\frac{1}{\pi} \int_{0}^{\infty} e^{-\theta^{\alpha}} \theta^{(n+1) \alpha-1} d \theta \int_{0}^{\infty} \psi\left(h \theta^{\alpha}-x\right) x^{n-j-(n+1) \alpha} d x .
$$

Evaluating the integrals we find

$$
\begin{aligned}
J_{n, j} & =(-1)^{n} \Gamma((n+1)(1-\alpha)-j)\left[\left(C_{n} E_{n}+D_{n} F_{n}\right)(\alpha \pi)\right]^{-1} \\
& =\frac{(-1)^{n} \Gamma((n+1)(1-\alpha)-j) n !}{\alpha \pi\left(1+h^{2}\right)^{(n+1) / 2}} \sin \left[(n+1)\left(\frac{\alpha \pi}{2}+\tan ^{-1}(h)\right)\right] .
\end{aligned}
$$

Hence $\gamma_{n}^{+}$is as claimed. If $(n-j) /(n+1)=\alpha$ then

$$
\int_{0}^{\infty} f^{(n-j)}(u) u^{n-(n+1) \alpha} d u=(-1)^{j+1} f^{(n-j-1)}(0) \Gamma((n+1)(1-\alpha)) .
$$

Evaluating $f^{(n-j-1)}(0)$ be using the inversion formula we find that $\gamma_{n}^{+}$is given as claimed.

Let $y=z x, 1 / k \leq z \leq k$. To establish (1.5a) we need to show that as $x \rightarrow \infty$, uniformly in $z$,

$$
[g(z x)-g(x)] x^{1-\alpha} \rightarrow\left[z^{\alpha-1}-1\right] \gamma_{0}^{+} .
$$

For this purpose note that

$$
g(z x)-g(x)=\int_{x}^{z x} d u \int_{0}^{\infty} f^{\prime}\left(t^{-1 / \alpha}(u+b t)\right) t^{-2 / \alpha} d t .
$$

Let $t^{-1 / \alpha} u=w$. Then

$$
g(z x)-g(x)=\int_{x}^{z x} d u u^{\alpha-2} \alpha \int_{0}^{\infty} f^{\prime}\left(w+b\left(\frac{w}{u}\right)^{1-\alpha}\right) w^{-\alpha+1} d w .
$$

Since $f^{\prime}$ is uniformly continuous and $w^{-\alpha+1}$ is integrable on $[0, A]$ for any finite $A$ it follows by dominated convergence that uniformly in $z \in[1 / k, k]$ as $x \rightarrow \infty$

$$
\begin{gathered}
x^{1-\alpha} \int_{x}^{z x} u^{\alpha-2} d u \int_{0}^{A} f^{\prime}\left(w+b\left(\frac{w}{u}\right)^{1-\alpha}\right) w^{-\alpha+1} d w \\
\rightarrow-\frac{\alpha}{1-\alpha}\left(z^{-\alpha+1}-1\right) \int_{0}^{A} f^{\prime}(w) w^{-\alpha+1} d w
\end{gathered}
$$


Fix $\varepsilon>0$. For some constant $k<\infty$ and all $u>0$

$$
\left|\int_{A}^{\infty} f^{\prime}\left(w+\left(\frac{w}{u}\right)^{1-\alpha} b\right) w^{1-\alpha} d w\right| \leq K A^{-2 \alpha} .
$$

We can thus choose $A$ such that $K A^{-2 \alpha} \leq \varepsilon$. But then uniformly in $z$

$$
\begin{aligned}
-\varepsilon\left(z^{-\alpha+1}-1\right) x^{-\alpha+1} & \leq \int_{x}^{z x} u^{\alpha-2} d u \int_{A}^{\infty} f^{\prime}\left(w+\left(\frac{w}{u}\right)^{1-\alpha} b\right) w^{1-\alpha} d w \\
& \leq \varepsilon\left(z^{1-\alpha}-1\right) x^{-\alpha+1} .
\end{aligned}
$$

Since

$$
-\frac{\alpha}{1-\alpha} \int_{0}^{A} f^{\prime}(w) w^{-\alpha+1} d w \rightarrow \frac{-\alpha}{1-\alpha} \int_{0}^{\infty} f^{\prime}(w) w^{-\alpha+1}=\gamma_{0}^{+}
$$

it follows from the above that $[g(z x)-g(x)] x^{1-\alpha} \rightarrow\left(z^{\alpha-1}-1\right) \gamma_{0}^{+}$uniformly in $z \in[1 / k, k]$. The argument for $[g(-z x)-g(-x)] x^{1-\alpha} \rightarrow \gamma_{0}^{-}$uniformly is essentially the same and will be omitted. Since both $\gamma_{0}^{+}$and $\gamma_{0}^{-}$are positive it follows from (1.5b) that $g(x)$ is asymptotically decreasing for $x>0$. It remains to show $g(x)$ is decreasing. For this purpose we write

$$
g(x)=\left(\frac{x}{b}\right)^{1-1 / \alpha} \int_{0}^{\infty} f\left(x^{1-1 / \alpha} b^{1 / \alpha} s^{-1 / \alpha}(s+1)\right) s^{-1 / \alpha} d s .
$$

Let $u=(s+1) s^{-1 / \alpha}$. Then $u$ is strictly decreasing on $(0, \infty)$ and maps $(0, \infty)$ onto $(0, \infty)$. Let $\psi$ be its inverse. Then

$$
g(x)=\frac{1}{b} \int_{0}^{\infty} f(z) \frac{\alpha \psi\left(z x^{1 / \alpha-1} b^{-1 / \alpha}\right)}{1+(1-\alpha) \psi\left(z x^{1 / \alpha-1} b^{-1 / \alpha}\right)} d z
$$

since $x^{1 / \alpha-1}$ is increasing and $\psi$ is decreasing the integrand is for each $z$ a decreasing function of $x$. Hence $g$ is decreasing.

Proof of Theorem 4. General theory [6, Proposition 11.2] shows that

$$
P_{x}\left(X_{W_{B^{-}}} \in d y\right)=g(y-x) \hat{\mu}_{B}(d y) .
$$

The results on $P_{x}\left(X_{W_{B}-} \in d y\right)$ follows at once from this fact and Theorems 2 and 3. Let $g_{B}(x, y)$ be the density of $\int_{0}^{\infty} P_{x}\left(T_{B}>t, X_{t} \in d y\right) d t$. General theory [6] shows

$$
g(y-x)-\int P_{x}\left(X_{T_{B}} \in d z\right) g(y-z)=g_{B}(x, y)
$$

and

$$
g(y-x)-\int \widehat{P}_{y}\left(X_{T_{B}} \in d z\right) g(z-x)=g_{B}(x, y) .
$$

Suppose $\beta<1$. Theorem 3 shows $g(y-x) \sim \tilde{g}(-x), x \rightarrow \infty$, uniformly in $y$ on compacts. Using this fact and $(2.15)$ we see that

$$
\lim _{x \rightarrow \infty} g_{B}(x, y) / \tilde{g}(-x)=\widehat{P}_{y}\left(T_{B}=\infty\right) .
$$


Now

$$
P_{x}\left(T_{B}<\infty\right)=\int g(y-x) \hat{\mu}_{B}(d y)
$$

Hence

$$
P_{x}\left(T_{B}<\infty\right) / \tilde{g}(-x) \rightarrow \hat{\mu}_{B}(\bar{B})=C(B)<\infty .
$$

Thus $\exists$ a sequence $\left\{x_{n}\right\}$ tending to $\infty \ni P_{x_{n}}\left(X_{T_{B}} \in d z\right) / \tilde{g}\left(-x_{n}\right)$ converges weakly to some measure $\pi(d z)$ supported on $\bar{B}$. Using (2.14) and (2.16) we find

$$
\int \pi(d z) g(y-z)=P_{y}\left(T_{B}<\infty\right) .
$$

The uniqueness of $\hat{\mu}_{B}$ now shows $\pi=\mu_{B}$. Consequently $P_{x}\left(X_{T_{B}} \in d z\right) / \tilde{g}(x)$ $\rightarrow \mu_{B}(d z)$ weakly. The same argument establishes the corresponding result for $x \rightarrow-\infty$ when $\beta>-1$. Finally the results for $\beta=1$ are obvious from the fact that the process moves continuously to the left.

Verification that (1.7) fails for processes with $\alpha<1$ and $\beta=1$. Suppose

$$
\begin{aligned}
\lim _{x \rightarrow-\infty} P_{x}\left(X_{T_{B}} \in d z \mid T_{B}<\infty\right) & =\lim _{x \rightarrow \infty} P_{x}\left(X_{T_{B}} \in d z \mid T_{B}<\infty\right) \\
& =\mu_{B}(d z) / C(B) .
\end{aligned}
$$

Let $d=\sup \{x: x \in B\}$. Then $\lim _{x \rightarrow \infty} P_{x}\left(X_{T_{B}} \in d z \mid T_{B}<\infty\right)=e_{d}(d z)$. Then $\lim _{x \rightarrow-\infty} P_{x}\left(X_{T_{B}} \in d y \mid T_{B}<\infty\right)=e_{d}(d y)$. Hence $C(B) e_{d}(d z)=\mu_{B}(d z)$. Thus

$$
\widehat{P}_{x}\left(T_{B}<\infty\right)=\int g(x-z) C(B) e_{d}(d z)=C(B) g(x-d) .
$$

Suppose $B$ is a finite interval $[a, d], a<d$. Then for $x \in(a, d)$, $\widehat{P}_{x}\left(T_{B}<\infty\right)=1$. Hence $1=C(B) g(x-d)$ for all $x \in(a, d)$. But that is impossible since $g(x-d)=e^{\gamma(x-d)}$ for $x<d$.

Verification that (1.15) fails for processes with $\alpha>1$ and $\beta=-1$. Let $B=[d, c], d<c$. Then as above we find for $d<x<c, 1=C(B) e^{-\gamma(x-d)}$, which is impossible.

Proof of Theorem 5. Observe that

$$
p(t, x)=t^{-1 / \alpha} f\left(t^{-1 / \alpha}(x+b t)\right) .
$$

The assertions about the asymptotic behavior of $p(t, x)$ follow at once from the above formula for $|\beta|<1$. For $\beta=1$ the above formula and Proposition 2.2 yield the desired asymptotic formula. General theory [6, Proposition 11.2] shows

$$
P_{x}\left(X_{W_{B^{-}}} \in d y\right)=g(y-x) \hat{\mu}_{B}(d y)
$$

Hence

$$
\begin{aligned}
P_{x}\left(W_{B}>t, X_{W_{B^{-}}} \in d y\right) & =\int p(t, z-x) g(y-z) \hat{\mu}_{B}(d y) d z \\
& =\int_{t}^{\infty}\left[p(s, y-x) \hat{\mu}_{B}(d y)\right] d s
\end{aligned}
$$


Thus

$$
P_{x}\left(W_{B} \in d t, X_{W_{B^{-}}} \in d y\right) / d t=p(t, y-x) \hat{\mu}_{B}(d y),
$$

from which (1.8) follows from the behavior of $p(t, x)$. The rest of the results in the theorem follow from the fact that $r(t)$ in [6] is here $\sim f(0) \alpha /(1-\alpha) t^{1-1 / \alpha}$ as $t \rightarrow \infty$ and results from the general theory [6, Theorems 14.1, 14.3].

Proof of Theorem 6. By Proposition 2.4

$$
g(x)=\sum_{j=1}^{n} A_{j} \int_{0}^{\infty} t^{-1 / \alpha}\left[t^{-1 / \alpha}(x+b t)\right]^{-(\alpha j+1)} d t+\varepsilon(x)
$$

where

$$
\varepsilon(x)=O\left(\int_{0}^{\infty} t^{-1 / \alpha}\left[(x+b t) t^{-1 / \alpha}\right]^{-[(n+1) \alpha+1]} d t\right)
$$

Since

$$
t^{-1 / \alpha}\left[t^{-1 / \alpha}(x+b t)\right]^{-(\alpha j+1)}=t^{j}(x+b t)^{-(\alpha j+1)}
$$

and

$$
\int_{0}^{\infty} s^{j}(1+s)^{-(\alpha j+1)} d s=\frac{1}{j+1}\left(\begin{array}{c}
\alpha j \\
j+1
\end{array}\right)^{-1},
$$

we find (1.11) holds by making the change of variable $t=(x / b) s$ in all the integrals.

If $\beta=-1$ then $\alpha p=1$ and the same computations used to establish Theorem 2 show that (1.12) holds. Now

$$
g(0)=\int_{0}^{\infty} t^{-1 / \alpha} f\left(t^{1-1 / \alpha} b\right) d t=\alpha p[(\alpha-1) b]^{-1} .
$$

If $\beta=1$ the process can go to the left only in a continuous manner. Since $X_{t} \rightarrow-\infty$ with probability one it must be that $h(-x)=1$ for all $x<0$. Since $h(x)=g(x) / g(0)$ and here $g(0)=1 / b$ it follows that for $x \leq 0, g(x)=1 / b$. To establish the monotonicity of $g(x)$ for $x>0$ we proceed as follows. Let $a \leq 0$ and $c>0$ and let $T$ be the first hitting time of the two point set $\{a, c\}$. Then

$$
\begin{aligned}
& h(c)=P_{0}\left(X_{T}=a\right) g(c-a)+P_{0}\left(X_{t}=c\right), \\
& h(a)=P_{0}\left(X_{T}=a\right)+P_{0}\left(X_{T}=c\right) g(a-c) .
\end{aligned}
$$

Solving for $P_{0}\left(X_{T}=a\right)$ and using $h(x)=g(x) / g(0)$ we find

$$
\begin{aligned}
P_{0}\left(X_{T}=a\right) & =\frac{g(a) g(0)-g(c) g(a-c)}{g(0)^{2}-g(a-c) g(c-a)} \\
& =\frac{g(0)^{2}-g(c) g(0)}{g(0)^{2}-g(0) g(c-a)}
\end{aligned}
$$

thus $g(0)^{2}-g(0) g(c-a) \geq g(0)^{2}-g(c) g(0)$. Hence $g(c-a) \leq g(c)$. Thus $g(x)$ is decreasing for $x>0$. 
To establish (1.13) we proceed as follows. Observe that for $x>0$,

$$
g(-x)=\left(\frac{x}{b}\right)^{1-1 / \alpha} \int_{0}^{\infty} s^{-1 / \alpha} f\left(x^{1-1 / \alpha} b^{1 / \alpha} s^{-1 / \alpha}(s-1)\right) d s .
$$

The function $u=s^{-1 / \alpha}(s-1)$ is strictly increasing and has strictly increasing inverse $\psi(u)=s$ for $u \in(-\infty, \infty)$. Making the change of variable to $u$ in the above integral we find

$$
g(-x)=\left(\frac{x}{b}\right)^{1-1 / \alpha} \int_{-\infty}^{\infty} f\left(u x^{1-1 / \alpha} b^{1 / \alpha}\right) \frac{\alpha \psi(u)}{1+(\alpha-1) \psi(u)} d u
$$

Now

$$
\frac{1}{b}=\int_{-\infty}^{\infty}\left(\frac{x}{b}\right)^{1-1 / \alpha} f\left(u x^{1-1 / \alpha} b^{1 / \alpha}\right) d u
$$

Hence

$$
g(-x)-\frac{1}{b}=\left(\frac{x}{b}\right)^{1-1 / \alpha} \int_{-\infty}^{\infty} f\left(u x^{1-1 / \alpha} b^{1 / \alpha}\right) \frac{\psi(u)-1}{1+(\alpha-1) \psi(u)} d u
$$

Let

$$
h(u)=\frac{\psi(u)-1}{1+(1-\alpha) \psi(u)} .
$$

Then $h(0)=0$ and $h^{\prime}(0)=1 / \alpha$. Decompose the integral on the RHS of $(2.17)$ into three integrals $I_{1}=\int_{c}^{\infty}, I_{2}=\int_{-\infty}^{-a}$ and $I_{3}=\int_{-a}^{c}$, where $a>0$ and $c>0$. Using Proposition 2.1 we find

$$
\left(\frac{x}{b}\right)^{1-1 / \alpha} I_{1} \sim\left[A_{+} b^{-2} \int_{c}^{\infty} h(u) u^{-(\alpha+1)} d u\right] x^{-\alpha+1}, \quad x \rightarrow \infty,
$$

and

$$
\left(\frac{x}{b}\right)^{1-1 / \alpha} I_{2} \sim\left[A_{-} b^{-2} \int_{-\infty}^{-a} h(u)|u|^{-(\alpha+1)} d u\right] x^{-\alpha+1}
$$

Now

$$
\begin{aligned}
\left(\frac{x}{b}\right)^{1-1 / \alpha} I_{3}=\left(\frac{x}{b}\right)^{1-1 / \alpha} & {\left[\int_{-a}^{c} f\left(u x^{1-1 / \alpha} b^{1 / \alpha}\right) \frac{u}{\alpha} d u\right.} \\
& +\int_{-a}^{c} f\left(u x^{1-1 / \alpha} b^{1 / \alpha}\left(h(u)-\frac{u}{\alpha}\right) d u\right] .
\end{aligned}
$$

Observe that

$$
\begin{aligned}
& \left(\frac{x}{b}\right)^{1-1 / \alpha} \int_{-a}^{c} \frac{u}{\alpha} f\left(u x^{1-1 / \alpha} b^{1 / \alpha}\right) d u \\
& \quad=\left(\frac{x}{b}\right)^{1-1 / \alpha} b^{-(1+1 / \alpha)} \alpha^{-1} \int_{-x^{1-1 / \alpha} b^{1 / \alpha} a}^{x^{1-1 / \alpha} b^{1 / \alpha} a} z f(z) \\
& \quad=\alpha^{-1} x^{1-1 / \alpha} b^{-(1+\alpha / 2)}\left[\left(\int_{c x^{1-1 / \alpha} b^{1 / \alpha}}^{\infty}+\int_{-\infty}^{-x^{1-1 / a} b^{1 / \alpha} a}\right) z f(z) d z\right]
\end{aligned}
$$


Using Proposition 2.1 we find that as $x \rightarrow \infty$

$$
\begin{aligned}
x^{\alpha-1} & \left(\frac{x}{b}\right)^{1-1 / \alpha} \int_{-a}^{c} f\left(u x^{1-1 / \alpha} b^{1 / \alpha}\right) \frac{u}{\alpha} d u \\
& \rightarrow-\left[\alpha(\alpha-1) b^{2}\right]^{-1}\left[A_{+} c^{-\alpha+1}-A_{-} a^{-\alpha+1}\right] .
\end{aligned}
$$

Now Proposition 2.1 shows that as $x \rightarrow \infty$

$$
b^{-1+1 / \alpha} x^{1-1 / \alpha+\alpha-1} f\left(u x^{1-1 / \alpha} b^{1 / \alpha}\right)\left[h(u)-\frac{u}{\alpha}\right],
$$

converges to $A_{+} u^{-(\alpha+1)}[h(u)-u / \alpha]$ for $u \geq 0$ and to $A_{-}|u|^{-(\alpha+1)}[h(u)-u / \alpha]$ for $u<0$. Additionally Proposition 2.1 shows

$$
f\left(u x^{1-1 / \alpha} b^{1 / \alpha}\right) x^{1-1 / \alpha+\alpha-1} \leq k_{0}|u|^{-(1+1 / \alpha)} b^{-(1+1 / \alpha)} .
$$

Since for some $k<\infty,|h(u)-u / \alpha| \leq k u^{2}$, we can conclude via dominated convergence that

$$
\begin{gathered}
\lim _{x \rightarrow \infty} b^{2} x^{\alpha-1}\left(\frac{x}{b}\right)^{1-1 / \alpha} \int_{0}^{c} f\left(u x^{1-1 / \alpha} b^{1 / \alpha}\right)\left[h(u)-\frac{u}{\alpha}\right] d u \\
=A_{+} \int_{0}^{c} u^{-(\alpha+1)}\left[h(u)-\frac{u}{\alpha}\right] d u
\end{gathered}
$$

and

$$
\begin{gathered}
\lim _{x \rightarrow \infty} b^{2} x^{\alpha-1}\left(\frac{x}{b}\right)^{1-1 / \alpha} \int_{-a}^{0} f\left(u x^{1-1 / \alpha} b^{1 / \alpha}\right)\left[h(u)-\frac{u}{\alpha}\right] d u \\
=A_{-} \int_{-a}^{0}|u|^{-(\alpha+1)}\left[h(u)-\frac{u}{\alpha}\right] d u .
\end{gathered}
$$

Let

and

$$
J_{+}(c)=\int_{0}^{c} u^{-(\alpha+1)}\left[h(u)-\frac{u}{\alpha}\right] d u
$$

Then (2.17)-(2.22) show

$$
J_{-}(a)=\int_{-a}^{0}|u|^{-(\alpha+1)}\left[h(u)-\frac{u}{\alpha}\right] .
$$

where for

$$
\lim _{x \rightarrow \infty}\left[g(-x)-\frac{1}{b}\right] x^{\alpha-1}=D b^{-2}
$$

$$
\begin{aligned}
K_{+} & =\int_{c}^{\infty} h(u) u^{-(\alpha+1)} d u-\frac{1}{\alpha(\alpha-1)} c^{-\alpha+1}+J_{+}(c) \\
& =\int_{0}^{\infty}\left[h(u)-\frac{u}{\alpha}\right] u^{-(\alpha+1)} d u
\end{aligned}
$$

and

$$
\begin{aligned}
K_{-} & =\int_{-\infty}^{-a} h(u)|u|^{-(\alpha+1)} d u+\frac{1}{\alpha(\alpha-1)} a^{-\alpha+1}+J_{-}(a) \\
& =\int_{-\infty}^{0}\left[h(u)-\frac{u}{\alpha}\right]|u|^{-(\alpha+1)} d u
\end{aligned}
$$


we have

$$
D=A_{+} K_{+}+A_{-} K_{-} .
$$

To evaluate $D$ note that $g(-x)-1 / b \equiv 0$ for $\beta=1$, and hence $D(\alpha, 1)=0$. Since $A_{-}(\alpha, 1)=0$ and $A_{+}(\alpha, 1)>0$ we can conclude from $(2.25)$ that $K_{+}=$ 0 . Since the value of $D$ does not depend on $a$ and $J_{-}(a) \rightarrow 0$ as $a \rightarrow 0$ we find that

$$
D=\lim _{a \rightarrow 0} A_{-}\left[\int_{-\infty}^{-a} h(u)|u|^{-(\alpha+1)} d u-\frac{1}{\alpha(\alpha-1)} a^{-\alpha+1}\right] .
$$

To evaluate the limit observe that

$$
\int_{-\infty}^{-a} h(u)|u|^{-(\alpha+1)} d u=\frac{1}{\alpha(\alpha-1)}[1-(1-\psi(-a))]^{-\alpha+1} .
$$

Taking $a=s^{-1 / \alpha}(s-1)$ we find

$$
\begin{aligned}
D & =\lim _{s \uparrow 1} A_{-}\left[1-(1-s)^{-\alpha+1}+s^{1-1 / \alpha}(1-s)^{-\alpha+1}\right] / \alpha(\alpha-1) \\
& =A_{-} / \alpha(\alpha-1) .
\end{aligned}
$$

Putting everything together we find $\left[g(-x)-1 / b-A_{+}\left[(\alpha)(\alpha-1) b^{2}\right]^{-1}\right] x^{\alpha-1} \rightarrow 0$.

To obtain an estimate of the error $\varepsilon(x)$ in (1.13) let $\phi_{1}=(x / b)^{1-1 / \alpha}, \phi_{2}=$ $x^{1-1 / \alpha} b^{1 / \alpha}$, and $H(u)=h(u)-u / \alpha$. Then

$$
\varepsilon(x)=I_{1}+I_{2}+I_{3}+I_{4}+I_{5}
$$

where

$$
\begin{aligned}
& I_{1}=\phi_{1} \int_{c}^{\infty}\left[f\left(\phi_{2} u\right)-A_{+}\left(\phi_{2} u\right)^{-(\alpha+1)}\right] h(u) d u \\
& I_{2}=\phi_{1} \int_{-\infty}^{-a}\left[f\left(\phi_{2} u\right)-A_{-}\left|\phi_{2} u\right|^{-(\alpha+1)}\right] h(u) d u \\
& I_{3}=-\frac{\phi_{1}}{\alpha \phi_{2}^{2}}\left[\int_{c \phi_{2}}^{\infty}\left[f(u)-A_{+} u^{-(\alpha+1)}\right] d u+\int_{-\infty}^{-a \phi_{2}}\left[f(u)-A_{-}|u|^{-(\alpha+1)}\right] u d u,\right. \\
& I_{4}=\phi_{1} \int_{-a}^{0}\left[f\left(\phi_{2} u\right)-A_{-}\left|\phi_{2} u\right|^{-(\alpha+1)}\right] H(u) d u
\end{aligned}
$$

and

$$
I_{5}=\phi_{1} \int_{-a}^{0}\left[f\left(\phi_{2} u\right)-A_{+}\left(\phi_{2} u\right)^{-(\alpha+1)}\right] H(u) d u .
$$

Using the fact [Proposition 2.4] that for all $u$,

$$
\left.\left.\left|f(u)-A_{ \pm}\right| u\right|^{-(\alpha+1)}|\leq K| u\right|^{-(2 \alpha+1)}
$$

and that $\phi_{1} \phi_{2}^{-(2 \alpha+1)}=b^{-3} x^{2(1-\alpha)}$ it easily follows that $I_{1}+I_{2}+I_{3}=O\left(x^{2(1-\alpha)}\right)$. Consider $I_{5}$. We can write $I_{5}=J_{1}+J_{2}$ where

$$
J_{1}=\phi_{1} \int_{0}^{1 / \phi_{2}}\left[f\left(u \phi_{2}\right)-A_{+}\left(\phi_{2} u\right)^{-(\alpha+1)}\right] H(u) d u
$$


and

$$
J_{1}=\phi_{1} \int_{1 / \phi_{2}}^{c}\left[f\left(u \phi_{2}\right)-A_{+}\left(\phi_{2} u\right)^{-(\alpha+1)}\right] H(u) d u .
$$

Since $H(u) \sim H^{\prime \prime}(0) u^{2} / 2, u \rightarrow 0$, it follows that

$$
J_{1} \sim\left[\int_{0}^{1}\left[f(u)-A_{+} u^{-(\alpha+1)}\right] \frac{u^{2}}{2} H^{\prime \prime}(0) d u\right] \frac{\phi_{1}}{\phi_{2}^{2}}=D_{1} x^{2 / \alpha(1-\alpha)},
$$

where $D_{1}$ is some constant. Now

$$
\left|J_{2}\right| \leq \phi_{1} K \int_{1 / \phi_{2}}^{c} u^{-(2 \alpha+1)} H(u) d u
$$

Since $H(u) \leq D_{2} u^{3}$ for some constant $D_{2}$ it follows that $J_{2}=O\left(x^{2 / \alpha(1-\alpha)}\right)$. Thus $\varepsilon(x)=O\left(x^{2 / \alpha(1-\alpha)}\right)$.

To establish (1.14a) we proceed as follows. Let

$$
h(u)=t^{-1 / \alpha} f\left(t^{-1 / \alpha}(u x+b t)\right) .
$$

Then $h^{\prime}(u)=f^{\prime}\left(t^{-1 / \alpha}(u x+b t)\right) t^{-2 / \alpha} x$. Thus

$$
g(z x)-g(x)=x \int_{1}^{z} d u \int_{0}^{\infty} f^{\prime}\left(t^{-1 / \alpha}(u x+b t)\right) t^{-2 / \alpha} d t .
$$

Let $\phi_{1}(u, x)=x^{2(1-1 / \alpha)} b^{2 / \alpha-1} u^{1-2 / \alpha}$ and let $\phi_{2}(u, x)=x^{1-1 / \alpha} b^{1 / \alpha} u^{1-1 / \alpha}$. The change of variable $t=u x s / b$ shows

$$
g(z x)-g(x)=\int_{1}^{z} d u \phi_{1} \int_{0}^{\infty} f^{\prime}\left(\phi_{2}(s+1) s^{-1 / \alpha}\right) s^{-2 / \alpha} d s .
$$

Using the fact that as $v \rightarrow \infty, f^{\prime}(r v) v^{\alpha+2} \rightarrow-(\alpha+1) A_{+}$uniformly in $r$ for $r$ in a finite closed subinterval of $(0, \infty)$ we find that uniformly in $u$, $1 / k \leq u \leq k$,

$$
\begin{aligned}
& x^{\alpha-1} \phi_{1} f^{\prime}\left(\phi_{2}(s+1) s^{-1 / \alpha}\right) \\
& \quad \rightarrow-b^{-2}(a+1) A_{+} u^{-\alpha} \int_{0}^{\infty}\left[(s+1) s^{-1 / \alpha}\right]^{-(\alpha+2)} d s=-A_{+} b^{-2} \alpha^{-1} .
\end{aligned}
$$

Thus

$$
\int_{1}^{z} d u x^{\alpha-1} \phi_{1} \int_{0}^{\infty} f^{\prime}\left(\phi_{2} s^{-1 / \alpha}(1+s)\right) d s \rightarrow \frac{\left(z^{-\alpha+1}-1\right)}{b^{2} \alpha(\alpha-1)} A_{+}
$$

uniformly in $z \in[1 / k, k]$. Thus uniformly in $z$ as just stated

$$
g(z x)-g(x) \sim \frac{A_{+}}{b^{2}(\alpha)(\alpha-1)}\left(z^{-\alpha+1}-1\right) x^{-\alpha+1} .
$$

But this is the same as

$$
g(y)-g(x) \sim \frac{A_{+}}{b^{2}(\alpha)(\alpha-1)}\left[g^{-\alpha+1}-x^{-\alpha+1}\right], \quad x, y \rightarrow \infty,
$$


for $x, y \ni 1 / k \leq y / x \leq k$. Let $y>x$. Then by what we have just shown for $x>x_{0}$ for some $x_{0}<\infty$

$$
\frac{g(y)-g(x)}{y^{-\alpha+1}-x^{-\alpha+1}} \leq \frac{2 A_{+}}{\left(b^{2}(\alpha)(\alpha-1)\right.} .
$$

Hence $g(y)-g(x)<0$. This shows $g(x)$ is asymptotically strictly decreasing.

The computations needed to establish (1.14b) are a combination of those used to establish (1.13) and (1.14a). For this reason we will simply outline the main points. Let $z \in[1 / k, k]$. Then

$$
g(-z x)-g(-x)=-\int_{1}^{z} \phi_{1} d u \int_{0}^{\infty} s^{-2 / \alpha} f^{\prime}\left(s^{-1 / \alpha}(s-1) \phi_{2}\right) d s .
$$

Let $v=s^{-1 / \alpha}(s-1)$ and let $\psi$ be its inverse function. Set

$$
H(v)=\alpha \psi^{1-1 / \alpha}[1+(\alpha-1) \psi]^{-1} \text {. }
$$

Then

$$
g(-z x)-g(-x)=\int_{1}^{z} \phi_{1} d u \int_{-\infty}^{\infty} f^{\prime}\left(\phi_{2} v\right) H(v) d v .
$$

Decompose the integral on $v$ as follows.

$$
\begin{aligned}
& \int_{-\infty}^{-a} f^{\prime}\left(\phi_{2} v\right) H(v) d v+\int_{-a}^{c} f^{\prime}\left(\phi_{2} v\right)\left[1+\frac{H^{\prime \prime}(0)}{2} v^{2}\right] \\
& +\int_{c}^{\infty} f^{\prime}\left(\phi_{2} v\right) H(v) d v+\int_{-a}^{c}\left[H(v)-1-\frac{H^{\prime \prime}(0)}{2} v^{2}\right] d v .
\end{aligned}
$$

Analyzing each of these contributions we find that uniformly in $z \in[1 / k, k]$ as $x \rightarrow \infty$

$$
x^{\alpha-1}\left[g(-z x)-g(-x] \rightarrow\left(z^{-\alpha+1}-1\right) A_{-} / \alpha(\alpha-1) .\right.
$$

It follows from $(2.27)$ that $g(-x)$ is asymptotically strictly decreasing except for $\beta=1$.

Proof of Theorem 8. The asymptotic behavior of $p(t, x)$ follows at once from the formula

$$
p(t, x)=t^{-1 / \alpha} f\left(t^{-1 / \alpha}(x+b t)\right)
$$

and Propositions 2.1 and 2.3. The rest of Theorem 8 follows from this asymptotic behavior just as for Theorem 5 .

Proof of Theorem 9. The set $[a, \infty)$ is a transient corecurrent set. Proposition 10.2 of [6] shows that every such set has capacity $b$. Additionally, there is a cocapacitory measure $\hat{\mu}_{a}$ such that

$$
P_{x}\left(T_{[a, \infty)}<\infty\right)=\int f(y-x) \hat{\mu}_{a}(d y) .
$$

The measure $\hat{\mu}_{a}$ is supported on $[a, \infty)$ and has total mass the capacity $C([a, \infty))=b$. Fix $\varepsilon>0$. By $(1.11)$ there is an $a_{0}<\infty$ such that for 
$y>a_{0}, g(y-x) \leq(1+\varepsilon) k(y-x)^{(1-\alpha)}$, where $k=\left[\alpha(\alpha-1) b^{2}\right]^{-1}$. Thus for $a>a_{0}$,

$$
P_{x}\left(T_{[a, \infty)}<\infty\right) \leq(1+\varepsilon) k \int(y-x)^{1-\alpha} \mu_{a}(d y) \leq(1+\varepsilon) k(a-x)^{1-\alpha} b .
$$

Thus

$$
\varlimsup_{a \rightarrow \infty} P_{x}\left(T_{[a, \infty)}<\infty\right) a^{1-a} \leq k b
$$

Let $C(d)=C([a, a+d])$ be the capacity of $[a, a+d]$. Then by Proposition 8.7 of [6] $c(d) \uparrow b$ as $d \rightarrow \infty$. Then for a $a>a_{0}$,

$$
\begin{aligned}
P_{x}\left(T_{[a, \infty)}<\infty\right) & \geq P_{x}\left(T_{[a, a+d]}<\infty\right)=\int g(y-x) \hat{\mu}_{[a, a+d]}(d y) \\
& \geq(1-\varepsilon) k \int(y-x)^{1-\alpha} \hat{\mu}_{[a, a+d]}(d y) \\
& \geq(1-\varepsilon)(a+d-x)^{1-\alpha} k C(d) .
\end{aligned}
$$

Hence

$$
\lim _{a \rightarrow \infty} P_{x}\left(T_{[a, \infty)}<\infty\right) a^{\alpha-1} \geq(1-\varepsilon) k C(d)
$$

Consequently

$$
\varliminf_{a \rightarrow \infty} P_{x}\left(T_{[a, \infty)}<\infty\right) a^{\alpha-1} \geq k b
$$

(1.16) now follows from (2.28) and (2.29).

Proof of Theorem 10. Suppose $a>0$. The $r$ th moment of $N_{a}$ is

$$
\mathrm{E} N_{a}^{r}=r ! \int_{0}^{a} \cdots \int_{0}^{a} g\left(x_{1}\right) \cdots g\left(x_{r}-x_{r-1}\right) d x_{1} \cdots d x_{r} .
$$

Using the fact that $g$ is bounded, that $\sup _{x} g(x)|x|^{1-\alpha}=k<\infty$, that $\tilde{g}(a x)=$ $a^{\alpha-1} \tilde{g}(x)$ and the fact that $g(x) \sim \tilde{g}(x), x \rightarrow \pm \infty$, routine asymptotic arguments show that as $a \rightarrow \infty$

$$
\begin{aligned}
\int_{0}^{a} & \cdots \int_{0}^{a} g\left(x_{1}\right) \cdots g\left(x_{r}-x_{r-1}\right) d x_{1} \cdots d x_{r} \\
& \sim a^{\alpha r} \int_{0}^{1} \cdots \int_{0}^{1} \tilde{g}\left(x_{1}\right) \cdots \tilde{g}\left(x_{r}-x_{r-1}\right) d x_{1} \cdots d x_{r} .
\end{aligned}
$$

Let

$$
I_{r}=\int_{0}^{1} \cdots \int_{0}^{1} \tilde{g}\left(x_{1}\right) \cdots \tilde{g}\left(x_{r}-x_{r-1}\right) d x_{1} \cdots d x_{r} .
$$


Then as $\tilde{g}(x) \leq A|x|^{\alpha-1}$ we find

$$
\begin{aligned}
I_{r} & \leq A^{r} \int_{0}^{1} \cdots \int_{0}^{1}\left|x_{1}\right|^{\alpha-1} \cdots\left|x_{r}-x_{r-1}\right|^{\alpha-1} d x_{1} \cdots d x_{r} \\
& =A^{r} \int_{0}^{1} \cdots \int_{0}^{1}\left|x_{1}\right|^{\alpha-1} \cdots\left|x_{r-1}-x_{r-2}\right|^{\alpha-1} \\
& \times\left[\frac{x_{r-1}^{\alpha}}{\alpha}+\frac{\left(1-x_{r-1}\right)^{\alpha}}{\alpha}\right] d x_{1} \cdots d x_{r-1} \\
& \leq \frac{2}{\alpha} A^{r} \int_{0}^{1} \cdots \int_{0}^{1}\left|x_{1}\right|^{\alpha-1} \cdots\left|x_{r-1}-x_{r-2}\right|^{\alpha-1} d x_{1} \cdots d x_{r-1} .
\end{aligned}
$$

Thus

$$
I_{r} \leq\left(\frac{2}{\alpha}\right)^{r} A^{r}
$$

Hence

$$
\left(r ! I_{r}\right)^{-1 / 2 r} \geq\left(\frac{2 a}{\alpha}\right)^{-1 / 2}(r !)^{-1 / 2 r} .
$$

Thus $\sum_{r}\left(r ! I_{r}\right)^{-1 / 2 r}=\infty$. Consequently, Carlemen's criterion now shows that the moments $r ! I_{r}$ uniquely determine a distribution. Hence $N(a) / a^{\alpha}$ converge in distribution to a random variable having moments $r ! I_{r}$. The arguments for the asymptotic behavior of $N(-a)$ and $N(a)+N(-a)$ are similar and will be omitted.

Proof of Theorem 13. We can write

$$
g(x)-g(0)=x \int_{0}^{1} d z \int_{0}^{\infty} f^{\prime}\left(t^{-1 / \alpha}(x z+b t)\right) t^{-2 / \alpha} d t .
$$

Assume $x>0$. Making the change of variable $u=t^{-1 / \alpha} x z$ we find

$$
[g(x)-g(0)] x^{1-\alpha}=\alpha \int_{0}^{1} z^{\alpha-2} d z \int_{0}^{\infty} f^{\prime}\left(u+b u^{1-\alpha}(x z)^{\alpha-1}\right) u^{1-\alpha} d u .
$$

For all $x>0$ and $z \in[0,1]$ there are constants $k_{1}$ and $k_{2}$ such that

$$
\left|f^{\prime}\left(u+b u^{1-\alpha}(x z)^{\alpha-1}\right) u^{1-\alpha}\right| \leq k_{1} u^{1-\alpha} 1_{(0,1)}(u)+k_{2} u^{-2 \alpha-1} 1_{(1, \infty)}(u)
$$

which is integrable. Using dominated convergence we can now conclude that

$$
\lim _{x \downarrow 0} x^{1-\alpha}[g(x)-g(0)]=-I(\alpha, \beta)
$$

where

$$
-I(\alpha, \beta)=\frac{\alpha}{\alpha-1} \int_{0}^{\infty} f^{\prime}(u) u^{1-\alpha} d u .
$$

The constant $I(\alpha, \beta)$ can be computed by methods similar (but simpler) than those used to compute $\gamma_{n}^{+}$in Theorem 3. It turns out that

$$
I(\alpha, \beta)=\frac{1}{\pi(\alpha-1)} \Gamma(2-\alpha)\left(1+h^{2}\right)^{-1 / 2} \cos \left(\frac{\pi(\alpha-1)}{2}+\tan ^{-1}(h)\right) .
$$


Now

$$
\begin{aligned}
g(x) & -g(0)-x^{\alpha-1} I(\alpha, \beta) \\
& =\alpha x^{\alpha-1} \int_{0}^{1} z^{\alpha-2} d z \int_{0}^{\infty}\left[f^{\prime}\left(u+b\left(\frac{z x}{u}\right)^{\alpha-1}\right)-f^{\prime}(u)\right] u^{1-\alpha} d u .
\end{aligned}
$$

By the mean value theorem, for some $\Delta$ between $u$ and $u+b(x z / x)^{\alpha-1}$,

$$
\int_{1}^{\infty}\left[f^{\prime}\left(u+b\left(\frac{x z}{u}\right)^{\alpha-1}\right)-f^{\prime}(u)\right] u^{1-\alpha}=\int_{1}^{\infty} f^{\prime \prime}(\Delta) u^{2(1-\alpha)} d u .
$$

Using the fact that $f^{\prime \prime}$ is bounded, and the fact that $\left|f^{\prime \prime}(u)\right| \leq k u^{-(\alpha+3)}$ for some $k<\infty$ we can conclude by dominated convergence that

$$
\lim _{x \downarrow 0} \int_{1}^{\infty} f^{\prime \prime}(\Delta) u^{2(1-\alpha)} d u=\int_{1}^{\infty} f^{\prime \prime}(u) u^{2(1-\alpha)} d u
$$

If $\alpha<3 / 2, u^{2(1-\alpha)}$ is integrable on $[0,1]$. Thus dominated convergence shows that in this case

$$
\lim _{x \downarrow 0} \int_{0}^{1} f^{\prime \prime}(\Delta) u^{2(1-\alpha)} d u=\int_{0}^{1} f^{\prime \prime}(u) u^{2(1-\alpha)} d u
$$

It follows from these facts and (2.31) that for $\alpha<3 / 2$ as $x \downarrow 0$

$$
g(x)-g(0)-x^{\alpha-1} I(\alpha, \beta) \sim x^{2(\alpha-1)} \alpha \int_{0}^{1} b z^{2 \alpha-3} d z \int_{0}^{\infty} f^{\prime \prime}(u) u^{2(\alpha-1)} d u .
$$

If $3 / 2 \leq \alpha<2$ we cannot obtain so precise an estimate. Observe that for some $k_{1}<\infty$,

$$
\left|\int_{0}^{x}\left[f^{\prime}\left(u+b\left(\frac{z x}{u}\right)^{\alpha-1}\right)-f^{\prime}(u)\right] u^{1-\alpha} d u\right| \leq k_{1} x^{2-\alpha},
$$

and for some $k_{2}<\infty$,

$$
\begin{aligned}
& \left|\int_{x}^{1} f^{\prime}\left(u+b\left(\frac{z x}{u}\right)\right)-f^{\prime}(u) u^{1-\alpha} d u\right| \\
& \quad=\left|\int_{x}^{1} f^{\prime \prime}(\Delta)(z x)^{\alpha-1} u^{2(1-\alpha)} d u\right| \leq k_{2}(z x)^{\alpha-1} \int_{x}^{1} u^{2(1-\alpha)} d u \\
& \quad=k_{2}(z x)^{\alpha-1}\left[1-x^{2(1-\alpha)+1}\right] / 2(1-\alpha)+1=O\left(x^{2-\alpha}\right) .
\end{aligned}
$$

Thus

$$
\int_{0}^{1} z^{2-\alpha} d z \int_{0}^{1}\left[f^{\prime}\left(u+b\left(\frac{x z}{u}\right)^{\alpha-1}\right)-f^{\prime}(u)\right] u^{1-\alpha} d u=O\left(x^{2-\alpha}\right) .
$$

But then using (2.31)

$$
g(x)-g(0)-I(\alpha, \beta) x^{\alpha-1}=x^{\alpha-1} O\left(x^{2-\alpha}\right)=O(x) \text {. }
$$

This establishes (1.26a). The proof of $(1.26 \mathrm{~b})$ is the same. 
Proof of Theorem 14. The arguments needed for both parts are different. To establish $(1.27 \mathrm{a})$ note that $f^{\prime}(v)=-A(1+\alpha) v^{-(\alpha+2)}+\varepsilon(v)$ for all $v \geq 0$ where $|\varepsilon(v)| \leq k v^{-(2 \alpha+2)}$.

Now

$$
g(x)-g(0)=x \int_{0}^{1} d u \int_{0}^{\infty} f^{\prime}\left(t^{-1 / \alpha}(u x+b t)\right) t^{-2 / \alpha} d t .
$$

Let $t=(x / b) s$. Then

$$
g(x)-g(0)=\int_{0}^{1} d u \int_{0}^{\infty} f^{\prime}\left(s^{-1 / \alpha}(u+s)\right) \phi_{1} s^{-2 / \alpha} d s \phi_{2}
$$

where $\phi_{1}=x^{1-1 / \alpha} b^{1 / \alpha}, \phi_{2}=b^{2 / \alpha-1} x^{2(1-1 / \alpha)}$. Hence

$$
\begin{aligned}
g(x)-g(0)= & -\int_{0}^{1} d u A(1+\alpha) \int_{0}^{\infty} s(u+s)^{1(\alpha+2)} d s \phi_{1}^{-(\alpha+2)} \phi_{2} \\
& +O\left(\int_{0}^{1} d u \int_{0}^{\infty}(u+s)^{-(2 \alpha+2)} s^{2} d s \phi_{1}^{-(2 \alpha+2)} \phi_{2}\right) \\
= & -\frac{A}{b^{2} \alpha(1-\alpha)} x^{1-\alpha}+O\left(x^{2(1-\alpha)}\right) .
\end{aligned}
$$

The argument needed for $(1.27 \mathrm{~b})$ is different. Let $\phi_{1}=(z x b)^{1-1 / \alpha}$ and $\phi_{2}=(z x)^{1-1 / \alpha} b^{1 / \alpha}$. Observe that $\phi_{1} / \phi_{2}=1 / b$. We can write for $x>0$,

$$
g(-x)=\int_{0}^{\infty} \phi_{1} f\left(\phi_{2} s^{-1 / \alpha}(s-1)\right) s^{-1 / \alpha} d s .
$$

The function $v=s^{-1 / \alpha}(s-1)$ has a unique maximum at $s=1 /(1-\alpha)$ and is asymptotic to 0 as $s \rightarrow \infty$. Let $\psi_{1}$ be its inverse with values in $(0,1 /(1-\alpha))$ and $\psi_{2}$ its inverse with values in $(1 /(1-\alpha), \infty)$. Let

$$
\gamma(1 /(1-\alpha))^{-1 / \alpha}(1 /(1-\alpha)-1) .
$$

Then

$$
\begin{aligned}
g(-x)= & \phi_{1} \int_{-\infty}^{\gamma} f\left(\phi_{2} v\right)\left[\frac{\alpha \psi_{1}}{1-(1-\alpha) \psi_{1}}\right] d v \\
& +\phi_{1} \int_{0}^{\gamma} f\left(\phi_{2} v\right) \frac{\alpha \psi_{2}}{(1-\alpha) \psi_{2}-1} d v .
\end{aligned}
$$

Observe that $1 / b=\phi_{1} \int_{-\infty}^{\infty} f\left(\phi_{2} v\right) d v$ and $\alpha /(1-\alpha) \int_{0}^{\infty} \phi_{1} f\left(\phi_{2} v\right) d v=g(0)$. Since $g(0-)=1 / b+g(0)$ if follows from (2.33) that

$$
\begin{aligned}
g(-x)-g(0-)= & \phi_{1} \int_{-\infty}^{\gamma} f\left(\phi_{2} v\right)\left[\frac{\alpha \psi_{1}}{1-(1-\alpha) \psi_{1}}-1\right] d v \\
& +\phi_{1} \int_{0}^{\gamma} f\left(\phi_{2} v\right)\left[\frac{\alpha \psi_{2}}{(1-\alpha) \phi_{2}-1}-\frac{\alpha}{1-\alpha}\right] d v \\
& -\frac{\phi_{1}}{1-\alpha} \int_{\gamma}^{\infty} f\left(\phi_{2} v\right) d v
\end{aligned}
$$


In each of the first two integrals the expressions in [ ] is $O(v)$ for $v$ near 0 . Multiplying both sides of (2.34) by $x^{\alpha-1}$ and using the fact that $f(v) \sim$ $A_{+} v^{-(\alpha+1)}, v \rightarrow \infty$, and $f(v) \sim A_{-}|v|^{-(\alpha+1)}, v \rightarrow \infty$, we can argue (by dominated convergence in the first two integrals) that as $x \downarrow 0$

$$
\begin{aligned}
x^{\alpha-1}[g(-x)-g(0-)]= & b^{-2} A_{-} \int_{-\infty}^{0}|v|^{-(\alpha+1)}\left[\frac{\alpha \psi_{1}}{1-(1-\alpha) \psi_{1}}-1\right] d v \\
& +b^{-2} A_{+} \int_{0}^{\gamma} v^{-(\alpha+1)}\left[\left[\frac{\alpha \psi_{1}}{1-(1-\alpha) \psi_{1}}-1\right]\right. \\
& \left.+\left[\frac{\alpha \psi_{2}}{1-(1-\alpha) \psi_{2}}-\frac{\alpha}{1-\alpha}\right]\right] d v s-\frac{1}{(1-\alpha) \alpha} \gamma^{-\alpha} .
\end{aligned}
$$

The integrals can be evaluated by the change of variable $v=s^{-1 / \alpha}(s-1)$. Doing so shows

$$
\int_{\infty}^{0}|v|^{-(\alpha+1)}\left[\frac{\alpha \psi_{1}}{1-(1-\alpha) \psi_{1}}-1\right]=-\frac{1}{\alpha(1-\alpha)}
$$

and $\int_{0}^{\gamma}=1 /(1-\alpha) \alpha \gamma^{-\alpha}$. Thus the coefficient of $A_{+}$is 0 . This shows

$$
\lim _{x \rightarrow 0}[g(-x)-g(0-)] x^{\alpha-1}=-A_{-}\left[\alpha(1-\alpha) b^{2}\right]^{-1} .
$$

To approximate the error $\varepsilon(x)$ let $H_{1}=\alpha \psi_{1}\left[1-(1-\alpha) \psi_{1}\right]^{-1}$ and $H_{2}=$ $\alpha \psi_{2}\left[(1-\alpha) \psi_{2}-\alpha /(1-\alpha)\right]^{-1}$. Let $A_{-}(\alpha, \beta)=A(\alpha,-\beta)$. From (2.34) we see that

$$
\begin{aligned}
g(-x) & -g(0-)+A_{-}\left[(\alpha)(1-\alpha) b^{2}\right]^{-1} x^{1-\alpha} \\
= & \phi_{1} \int_{-\infty}^{0}\left[f\left(\phi_{2} v\right)-A_{-}\left|\phi_{2} v\right|^{-(\alpha+1)}\right] H_{1}(v) d v \\
& +\phi_{1} \int_{0}^{\gamma}\left[f\left(\phi_{2} v\right)-A\left(\phi_{2} v\right)^{-(\alpha+1)}\right] H(v) d v \\
& -\frac{\phi_{1}}{1-\alpha} \int_{\gamma}^{\infty}\left[f\left(\phi_{2} v\right)-A\left(\phi_{2} v\right)^{-(\alpha+1)}\right] d v .
\end{aligned}
$$

Suppose $\alpha<1 / 2$. Using Proposition 2.4 we can argue be dominated convergence that the RHS of (2.36) is asymptotic to

$$
\begin{gathered}
A_{2}(\alpha,-\beta) \phi_{1} \int_{-\infty}^{0}\left|\phi_{2} v\right|^{-(2 \alpha+1)} H_{1}(v) d v+A_{2} \phi_{1} \int_{0}^{\gamma}\left(\phi_{2} v\right)^{-(2 \alpha+1)} H_{2}(v) d v \\
-\frac{\phi_{1} A_{2}}{1-\alpha} \int_{\gamma}^{\infty}\left(\phi_{2} v\right)^{-(2 \alpha+1)} d v=k x^{2(1-\alpha)}
\end{gathered}
$$

where $k$ is some finite constant.

If $\alpha \geq 1 / 2$, then $v^{-2 \alpha}$ is not integrable near 0 and we cannot proceed as above. In this case we replace $\int_{-\infty}^{0}$ by $\int_{-\infty}^{-1}+\int_{-1}^{0}$ and $\int_{0}^{\gamma}$ by $\int_{0}^{1}+\int_{1}^{\gamma}$. As 
above, for some constant $k^{\prime}$ all terms except $\int_{-1}^{0}$ and $\int_{0}^{1}$ are $\sim k^{\prime} x^{2(1-\alpha)}$. Now

$$
\begin{aligned}
\phi_{1} \int_{0}^{1}[ & \left.f\left(\phi_{2} v\right)-A\left(\phi_{2} v\right)^{-(\alpha+1)}\right] H_{2}(v) d v \\
= & \phi_{1} \int_{0}^{1 / \phi_{2}}\left[f\left(\phi_{2} v\right)-A\left(\phi_{2} v\right)^{-(\alpha+1)}\right] H_{2}(v) d v \\
& +\phi_{1} \int_{1 / \phi_{2}}^{1}\left[f\left(\phi_{2} v\right)-A\left(\phi_{2} v\right)^{-(\alpha+1)}\right] H_{2}(v) d v .
\end{aligned}
$$

Making the change of variable $r \phi_{2}=u$ in the first integral and using the fact that $H(v) \sim H^{\prime}(0) v$ as $v \rightarrow 0$ we find

$\phi_{1} \int_{0}^{1 / \phi_{2}}\left[f\left(\phi_{2} v\right)-A\left(\phi_{2} v\right)^{-(\alpha+1)}\right] H_{2}(v) d v \sim \frac{1}{b \phi_{2}} \int_{0}^{1}\left[f(v)-A v^{-(\alpha+1)}\right] H_{2}(0) v d v$

It follows from Proposition 2.4 and the fact that $f$ is bounded and continuous that, for some $k<\infty,\left|f(v)-A v^{-(\alpha+1)}\right| \leq k v^{-(2 \alpha+1)}$ for all $v>0$. Thus for some constant $k^{\prime}<\infty$,

$$
\begin{aligned}
& \phi_{1}\left|\int_{1 / \phi_{2}}^{1}\left[f\left(\phi_{2} v\right)-A v^{-(\alpha+1)}\right] H(v) d v\right| \\
& \quad \leq \phi_{1} \phi_{2}^{-(2 \alpha+1)} k^{\prime} \int_{1 / \phi_{2}}^{1} v^{-2 \alpha} d v \\
& \quad=\phi_{1} \phi_{2}^{-(2 \alpha+1)} k^{\prime}\left[1-\phi_{2}^{2 \alpha}\right] /(-2 \alpha+1) \\
& \quad=\frac{1}{b} k^{\prime}(2 \alpha-1)^{-1}\left[\phi_{2}^{-1}-\phi_{2}^{-2 \alpha}\right]
\end{aligned}
$$

Thus for $\alpha \geq 1 / 2$,

$$
\phi_{1} \int_{0}^{1}\left[f\left(\phi_{2} v\right)-A\left(v^{-(\alpha+1)} \phi_{2}\right)\right] H(v) d v=O\left(x^{(1-\alpha) / \alpha)}\right) .
$$

Similarly,

$$
\phi_{1} \int_{-1}^{0}\left[f\left(\phi_{2} v\right)-A_{-}\left|\phi_{2} v\right|^{-(\alpha+1)}\right]=O\left(x^{(1-\alpha) / \alpha}\right) .
$$

Proof of Theorem 16. Let $h=\frac{2 \beta}{\pi}$. Suppose $a>0$. Then

$$
\begin{aligned}
\operatorname{E} N(a)^{r} & =r ! \int_{0}^{a} \cdots \int_{0}^{a} g\left(x_{1}\right) g\left(x_{2}-x_{1}\right) \cdots g\left(x_{r}-x_{r-1}\right) d x_{1}, \ldots, d x_{r} \\
& =r ! a^{r} \int_{0}^{1} \cdots \int_{0}^{1} g\left(a x_{1}\right) \cdots g\left(a\left(x_{r}-x_{r-1}\right)\right) d x_{1}, \ldots, d x_{r} .
\end{aligned}
$$

Routine Abelian arguments show that as $a \rightarrow \infty$

$$
\begin{gathered}
(\ln a)^{r} \int_{0}^{1} \cdots \int_{0}^{1} g\left(a x_{1}\right) \cdots g\left(a\left(x_{r}-x_{r-1}\right)\right) d x_{1} \cdots d x_{r} \\
\quad \rightarrow\left(\frac{1}{\pi h^{2}}\right)^{r} H\left(x_{1}, \ldots, x_{r}\right) d x_{1} \cdots d x_{r}
\end{gathered}
$$


where

$$
H\left(x_{1}, \ldots, x_{r}\right)=\left[1+\beta \operatorname{sgn}\left(x_{1}\right)\right] \cdots\left[1+\beta \operatorname{sgn}\left(x_{r}-x_{r-1}\right)\right] .
$$

Let

$$
\mu_{r}=r !\left(\frac{1}{\pi h^{2}}\right)^{r} \int_{0}^{1} \cdots \int_{0}^{1} H\left(x_{1}, \ldots, x_{r}\right) d x_{1} \cdots d x_{r} .
$$

Then as $a \rightarrow \infty$

$$
\mathrm{E}\left[N(a) \frac{\ln a}{a}\right] \rightarrow \mu_{r} .
$$

Carleman's criterion shows the $\left\{\mu_{r}\right\}$ uniquely determine the distribution of a random variable $N^{+}$having moments $\left\{\mu_{r}\right\}$. Thus $N(a) \frac{\ln a}{a} \stackrel{D}{\rightarrow} N^{+}$. In this case it is possible to determine the distribution of $N^{+}$. For this purpose we need to make use of some combinatorial facts due to Euler. These can be found in Volume 3 of the books of E. Knuth, The art of computer programming, §5.1.3. Let $\left\langle\begin{array}{l}r \\ j\end{array}\right\rangle$ be the Eulerian numbers. Suppose $x_{1}, \ldots, x_{r}$ are distinct real numbers. Let $\gamma_{r j}$ be the number of permutations of $x_{1}, \ldots, x_{r}$ such that $x_{i_{\nu}+1}-x_{i_{\nu}}>0$ exactly $j$ times. Then (by definition) $\gamma_{r j}=\left\langle\begin{array}{c}r \\ r-j\end{array}\right\rangle$. Let

$$
\phi_{r}(z)=\frac{1}{r !} \sum_{j=0}^{r}\left\langle\begin{array}{l}
r \\
j
\end{array}\right\rangle z^{j} \quad \text { and } \phi(z, x)=\sum_{r=0}^{\infty} \phi_{r}(z) x^{r} .
$$

Then Euler's result is

$$
\phi(z, x)=z(1-z)\left[e^{(z-1) x}-z\right]^{-1} .
$$

Let $G$ be the set of $r$ ! permutations and $S$ the simplex $\left\{0<x_{1}<\cdots<x_{r}<\right.$ 1 ). Since $S_{1}$ has volume $(r !)^{-1}$ we see that

$$
\begin{aligned}
\int_{0}^{1} & \ldots \int_{0}^{1} H\left(x_{1}, \ldots, x_{r}\right) d x_{1} \cdots d x_{r} \\
& =\sum_{\tau \in G} \int \ldots \int H\left(\tau\left(x_{1}, \ldots, x_{r}\right)\right) d x_{1} \cdots d x_{r} \\
& =\sum_{j=0}^{r-1} \gamma_{r j}(1+\beta)^{j+1}(1-\beta)^{r-j-1}[r !]^{-1} .
\end{aligned}
$$

Let $\beta=p-q$ where $1+\beta=2 p$ and $1-\beta=2 q$. Using the above we find

$$
\mu_{r}=\sum_{j=1}^{r}\left\langle\begin{array}{l}
r \\
j
\end{array}\right\rangle\left(\frac{2}{\pi h^{2}}\right)^{2} \frac{p^{r+1}}{q}\left(\frac{q}{p}\right)^{j}=\frac{p}{q}\left(\frac{2 p}{\pi h^{2}}\right)^{r} r ! \phi_{r}\left(\frac{p}{q}\right) .
$$

The moment generating function of $\left\{\mu_{r}\right\}$ is

$$
M=\sum_{r=0}^{\infty} \mu_{r} \frac{s^{r}}{r !}=\frac{p}{q} \sum_{r=0}^{\infty}\left(\frac{2 p s}{\pi h^{2}}\right)^{r} \phi_{r}\left(\frac{q}{p}\right)=\frac{p}{q} \Phi\left(\frac{q}{p}, \frac{2 p s}{\pi h^{2}}\right) .
$$

Using (2.37) we find

$$
M(s)=(p-q)\left[p e^{-s / h}-q\right]
$$


If $\beta>0$ then $p>q$ and we can write

$$
M(s)=\left(1-\frac{q}{p}\right) e^{s / h}\left[1-\frac{q}{p} e^{s / h}\right]^{-1}, \quad p>q .
$$

If $p<q$ we write

$$
M(s)=\left[1-\frac{p}{q}\right]\left[1-\frac{p}{q} e^{s / h}\right]^{-1}, \quad p<q .
$$

Now (2.39) is the moment generating function of $1 /|h|(Z+1)$ where $Z$ is geometrically distributed with parameter $q / p$ while $(2.40)$ is the moment generating function of $Z / h$ where $Z^{\prime}$ is geometrically distributed with parameter $p / q$. This establishes the distribution of $N^{+}$. Since $-X_{t}$ is the Cauchy process $x_{t}$ with $\beta$ replaced by $-\beta$ the distribution of $N^{-}$also follows from the above results. The proof of the asymptotic distribution of $N(a)+N(-a)$ is essentially the same and will be omitted.

\section{APPENDIX}

Let $X_{t}$ be a Levy process on $R$ starting at 0 with $\log$ characteristic function $-t \psi(\theta)$, where

$$
\psi(\theta)=\int_{R}\left(1-e^{i \theta y}\right) M(d y)+i b \theta
$$

such that $b>0$ and $\int_{|y| \leq 1}|y| M(d y)<\infty$.

Let $\lambda>0$ and let $G^{\lambda}(A)=E \int 1_{A}\left(X_{t}\right) e^{-\lambda t} d t$. General theory [3] yields the following facts. $G^{\lambda}(d x) \ll d x$, and there is a version $g^{\lambda}(x)$ for the density having the following properties.

(i) $g^{\lambda}$ is bounded and continuous for all $x \neq 0$.

(ii) At $0 g^{\lambda}$ has a jump, $g^{\lambda}(0+)=g^{\lambda}(0)$ and $g^{\lambda}(0-)>g^{\lambda}(0)$.

If the process is transient these facts hold for $\lambda=0$.

Theorem A. Let $\lambda>0$. Then

$$
g^{\lambda}(0-)-g^{\lambda}(0)=\frac{1}{b} .
$$

If the process is transient (3.1) holds for $\lambda=0$.

Proof. Let

$$
I(\theta)=\int_{R} \sin (\theta y) M(d y) \text { and } J(\theta)=\int_{R}[1-\cos (\theta y)] M(d y) .
$$

Then

$$
\operatorname{Re}(\lambda+\psi)=\lambda+J, \quad \operatorname{Im}(\lambda+\psi)=b \theta-I .
$$

Observe that $J \geq 0$. We will now show

$$
\lim _{|\theta| \rightarrow \infty}\left|\frac{I(\theta)}{\theta}\right|=0
$$


and

$$
\lim _{|\theta| \rightarrow \infty}\left|\frac{J(\theta)}{\theta}\right|=0 \text {. }
$$

To this end let $k=\sup _{z}|\sin (z) / z|$. Fix $\varepsilon>0$. We can then choose $\delta>0$ such that

$$
k \int_{|z| \leq \delta}|z| M(d z) \leq \varepsilon
$$

Now

$$
\begin{aligned}
\left|\frac{I(\theta)}{\theta}\right| & \leq \int_{|z| \leq \delta}\left|\frac{\sin (z \theta)}{z \theta}\right||z| M(d z)+\frac{1}{|\theta|} \int_{|z| \geq \delta}|\sin (z \theta)| M(d z) \\
& \leq \varepsilon+\frac{1}{|\theta|} M[|z|>\delta] .
\end{aligned}
$$

Thus (3.2) holds. An analogous argument shows (3.3) holds. Now

$$
|\theta||\lambda+\psi|=|\theta|^{2}\left[\left(\frac{\lambda}{\theta}+\frac{J}{\theta}\right)^{2}+\left(b-\frac{I(\theta)}{\theta}\right)^{2}\right] \text {. }
$$

Hence for some $N$

$$
|\theta||\lambda+\psi| \geq \frac{b}{2} \theta^{2}
$$

for all $|\theta|>N$. Since $|\lambda+\psi| \geq \lambda$ it follows that for any $h>0$,

is integrable over $R$.

$$
\frac{1-\cos (\theta h)}{\theta h(\lambda+\psi)}
$$

Let $Z$ have the standard normal distribution and be independent of $\left\{X_{t}\right\}$. Let $Y_{t}(\sigma)=X_{t}+\sigma Z$ for $\sigma>0$. Then

$$
\frac{1}{h} P\left(0 \leq Y_{t}(\sigma) \leq h\right)=\frac{1}{2 \pi} \int_{R} e^{-t \psi(\theta)} e^{-\sigma^{2} \theta^{2} / 2}\left[\left(1-e^{-i \theta h}\right) / i \theta h\right] d \theta
$$

and

$$
\frac{1}{h} P\left(-h \leq Y_{t}(\sigma) \leq 0\right)=\frac{1}{2 \pi} \int_{R} e^{-t \psi(\theta)} e^{-\sigma^{2} \theta^{2} / 2}\left[\left(e^{i \theta h}-1\right) / i \theta h\right] d \theta
$$

Hence

$$
\begin{aligned}
\frac{1}{h}[P(-h & \left.\left.\leq Y_{t}(\sigma) \leq 0\right)-P\left(0 \leq Y_{t}(\sigma) \leq h\right)\right] \\
& =\frac{1}{\pi} \int_{R} e^{-t \psi(\theta)} e^{-\theta^{2} \sigma^{2} / 2}[(\cos (\theta h)-1) / i \theta h] d \theta .
\end{aligned}
$$

Multiply both sides by $e^{-\lambda t}$ for $\lambda>0$ and then integrate over $t$ on $(0, \infty)$. Since $\int_{R} \int_{0}^{\infty} e^{-\lambda t} e^{-\theta^{2} \sigma^{2} / 2} d \theta d t<\infty$ it follows by Fubini's theorem that

$$
\begin{gathered}
\frac{1}{h} \int_{0}^{\infty} e^{-\lambda t}\left[P\left(-h \leq Y_{t}(\sigma) \leq 0\right)-P\left(0 \leq Y_{t}(\sigma) \leq h\right] d t\right. \\
=\frac{1}{\pi} \int i \frac{1-\cos (\theta h)}{\theta h[\lambda+\psi]} e^{-\theta^{2} \sigma^{2} / 2} d \theta
\end{gathered}
$$


Since $(1-\cos (\theta h)) /(\theta h|\lambda+\psi|)$ is integrable, we can let $\sigma \downarrow 0$ and conclude via dominated convergence that

$$
\begin{aligned}
\frac{1}{h} \int_{0}^{\infty} & e^{-\lambda t}\left[P\left(-h \leq x_{t} \leq 0\right)-P\left(0 \leq X_{t} \leq h\right)\right] d t \\
& =\operatorname{Re} \frac{1}{\pi} \int \frac{i[1-\cos (\theta h)]}{\theta h(\lambda+\psi)} d \theta \\
& =\frac{2}{\pi} \int_{0}^{\infty} \frac{1-\cos (\theta h)[b \theta-I]}{\theta h\left((\lambda+J)^{2}+(b \theta-I)^{2}\right.} d \theta \\
& =\frac{2}{\pi} \int_{0}^{\infty} \frac{1-\cos (\theta h)(1-I / b \theta)}{\theta^{2} h b\left[(\lambda / \theta b+J / b \theta)^{2}+(1-I / b \theta)^{2}\right]} d \theta .
\end{aligned}
$$

Now the extreme LHS of (3.7) is

$$
\frac{1}{h} \int_{0}^{h}\left[g^{\lambda}(-x)-g^{\lambda}(x)\right] d x \text {. }
$$

Let

$$
H(u)=\left(1-\frac{I(u)}{u}\right)\left[\left(\frac{\lambda}{u b}+J(u)\right)^{2}+\left(1-\frac{J(u)}{b u}\right)^{2}\right]^{-1} .
$$

The change of variable $\theta h=z$ on the extreme RHS of (3.7) shows

$$
\frac{1}{h} \int_{0}^{h}\left[g^{\lambda}(-x)-g^{\lambda}(x)\right] d x=\frac{2}{\pi} \int_{0}^{\infty} \frac{1-\cos (z)}{b z^{2}} H\left(\frac{z}{h}\right) d z .
$$

Since $H(u)$ is bounded and converges to 1 as $u \rightarrow \infty$ we can conclude by dominated convergence that

$$
\lim _{h \downarrow 0} \frac{1}{h} \int_{0}^{h}\left[g^{\lambda}(-x)-g^{\lambda}(x)\right] d x=\frac{2}{\pi} \int_{0}^{\infty} \frac{1-\cos (z)}{b z^{2}} d z=\frac{1}{b} .
$$

Thus (3.1) holds.

If the process is transient let $g(x)=g^{0}(x)$. Since $g^{\lambda}(x) \uparrow g(x)$ as $\lambda \downarrow 0$ it follows that $g^{\lambda}(0) \rightarrow g(0)$ as $\lambda \downarrow 0$. Now $g^{\lambda}(-x) \leq g^{\lambda}(0-)$. Hence $g(-x) \leq$ $\underline{\lim }_{\lambda \downarrow 0} g^{\lambda}(0)$ and letting $x \downarrow 0$ we find $g(0-) \leq \underline{\lim }_{\lambda \downarrow 0} g^{\lambda}(0)$. But $g^{\lambda}(-x) \leq$ $g(-x)$ so letting $x \downarrow 0$ we find $g^{\lambda}\left(0_{-}\right) \leq g\left(0_{-}\right)$. Thus $g^{\lambda}\left(0_{-}\right) \rightarrow g\left(0_{-}\right)$as $\lambda \downarrow 0$. Hence $(3.1)$ holds for $\lambda=0$ in the transient case.

\section{REFERENCES}

1. R. M. Blumenthal and R. K. Getoor, Local times for Markov processes, Z. Wahrsch. Verw. Gebiete 3 (1964), 50-74.

2. L. Breiman, On some limit theorems similar to the arc sin law, Theor. Probab. Appl. 10 (1965), 323-331.

3. J. Bretagnolle, Résultats de Kesten sur les processus a' accroisements independantes, Séminaire de Probabilités V, Lecture Notes in Math., vol. 191, Springer-Verlag, Berlin and New York, 1971, pp. 21-36.

4. B. Fristed, Sample functions of processes with stationary, independent increments, Advances in Probability III, Marcel Dekker, New York, 1974, pp. 241-396. 
5. I. A. Ibragimov and Yu. V. Linnik, Independent and stationary random variables.

6. S. C. Ports and C. J. Stone, Infinitely divisible processes and their potential theory. I, II, Ann. Inst. Fourier (Grenoble) 21 (1971), 157-275; 179-265.

7. __ The asymmetric Cauchy processes on the line, Ann. Math. Statist. 40 (1969), 137-143.

8. S. C. Port, Occupation time and Lebesgue measure of the range of a Levy processes, Proc. Amer. Math. Soc. 103 (1988), 1241-1248.

9. W. E. Pruitt and S. J. Taylor, The behavior of asymmetric Cauchy processes for large time, Ann. Probab. 11 (1983), 302-327.

10. A. V. Skorohod, Asymptotic formulas for stable distribution laws, Selected Transl. Math. Stat. Probab., vol. 1, Amer. Math. Soc., Providence, R. I., 1961, pp. 157-161.

Department of Mathematics, University of California, Los Angeles, California 90024 